\title{
Multiobjective Optimization and Evolutionary Algorithms for the Application Mapping Problem in Multiprocessor System-on-Chip Design
}

\author{
Cagkan Erbas, Selin Cerav-Erbas, and Andy D. Pimentel
}

\begin{abstract}
Sesame is a software framework that aims at developing a modeling and simulation environment for the efficient design space exploration of heterogeneous embedded systems. Since Sesame recognizes separate application and architecture models within a single system simulation, it needs an explicit mapping step to relate these models for cosimulation. The design tradeoffs during the mapping stage, namely, the processing time, power consumption, and architecture cost, are captured by a multiobjective nonlinear mixed integer program. This paper aims at investigating the performance of multiobjective evolutionary algorithms (MOEAs) on solving large instances of the mapping problem. With two comparative case studies, it is shown that MOEAs provide the designer with a highly accurate set of solutions in a reasonable amount of time. Additionally, analyses for different crossover types, mutation usage, and repair strategies for the purpose of constraints handling are carried out. Finally, a number of multiobjective optimization results are simulated for verification.
\end{abstract}

Index Terms-Design space exploration, evolutionary algorithms, mixed integer programming, multiobjective optimization, multiprocessor system-on-chip (SoC) design.

\section{INTRODUCTION}

$\mathbf{M}$ ODERN embedded systems come with contradictory design constraints. On one hand, these systems target mass production and battery-based devices, and therefore should be cheap and power efficient. On the other hand, they still need to show high (sometimes real-time) performance, and often support multiple applications and standards which requires high programmability. This wide spectrum of design requirements leads to complex system-on-chip (SoC) architectures, consisting of several types of processors from fully programmable microprocessors to configurable processing cores and customized hardware components. The ensuant high complexity of embedded systems design has led to a new design paradigm, known as the system-level design [1] which has the following two important ingredients:

- using a platform architecture that is shared among multiple applications, rather than designing one for each of them;

Manuscript received September 14, 2004; revised April 1, 2005. This work was supported in part by PROGRESS, the embedded systems research program of the Dutch organization for scientific research NWO, the Dutch Ministry of Economic Affairs, and the technology foundation STW.

C. Erbas and A. Pimentel are with the Department of Computer Science, University of Amsterdam, 1098 SJ Amsterdam, The Netherlands (e-mail: cagkan@ science.uva.nl; andy@science.uva.nl).

S. Cerav-Erbas is with the School of Management, Université Catholique de Louvain, 1348 Louvain-la-Neuve, Belgium (e-mail: cerav@ poms.ucl.ac.be).

Digital Object Identifier 10.1109/TEVC.2005.860766
- starting modeling and exploration with abstract executable components and gradually lowering the abstraction level by inserting more implementation details in every step, with the intention of reaching an optimal SoC architecture.

In system-level design, early exploration of the design space plays a crucial role as it allows evaluate of different architecture alternatives without the burden of low-level primitives. In terms of design time, it would otherwise be impossible to evaluate several alternatives, if one started at a lower level which requires the synthesis and verification of different parts of the design. The models at the system-level normally capture the behavior of the application, characteristics of the architecture, and the various relations between the application and the architecture, such as the allocation (which components of the platform architecture are used), the binding (mapping of application processes onto architecture resources), or the scheduling (execution order of processes). The analytical and simulation models synthesized at the system-level can provide reasonable estimations of performance [2], power consumption [3], or cost of the design [4], while minimizing the requirements in terms of modeling effort and simulation time that is needed in the early design stages.

The Sesame framework ${ }^{1}[5]$, [6], which we develop within the context of the Artemis project [7], provides methods and tools for the efficient design space exploration of heterogeneous embedded multimedia systems. Using Sesame, a designer can model embedded applications and $\mathrm{SoC}$ architectures at the system-level, map the former onto the latter using evolutionary optimizers which consider multiple design objectives simultaneously, and perform application-architecture cosimulations for rapid performance evaluations. Based on these evaluations, the designer can further refine (parts of) the design, experiment with different hardware/software partitionings, perform cosimulations at multiple levels of abstraction, or mixed level cosimulations where architecture model components operate at different levels of abstraction. To achieve this flexibility, the Sesame environment recognizes separate application and architecture models within a single system simulation. The application model defines the functional behavior of an application, including both computation and communication behaviors. The architecture model defines architecture resources and captures their performance constraints. An explicit mapping step maps an application model onto an architecture model for cosimulation.

${ }^{1}$ http://sesamesim.sourceforge.net. 
Until recently, the mapping step in Sesame was assumed to be performed by an experienced designer, intuitively. However, this assumption was increasingly becoming inappropriate for efficient design space exploration. First of all, the Sesame environment targets exploration at an early design stage where the design space is very large. At this stage, it is very hard to make critical decisions such as mapping without using any analytical method or a design tool, since these decisions seriously affect the rest of the design process, and in turn, the success of the final design. Besides, modern embedded systems are already quite complicated, generally having a heterogeneous combination of hardware and software parts possibly with dynamic behavior. It is also very likely that these embedded systems will become even more complex in the near future, and intuitive mapping decisions will eventually become obsolete for future designs. Moreover, coping with the design constraints of embedded systems, there exist multiple criteria to consider, like the processing times, power consumption and cost of the architecture, all of which further complicate the mapping decision.

In Sesame, these issues are captured by means of a multiobjective combinatorial optimization problem [8]. Due to its large size and nonlinear nature, it is realized that the integration of a fast and accurate optimizer is of crucial importance for this problem. The primary aim of the multiobjective optimization process is to provide the designer with a set of tradable solutions, rather than a single optimal point. The evolutionary algorithms (EAs), in general, seem to be the best choice for attacking such problems, as they evolve over a population rather than a single solution. For this reason, numerous multiobjective evolutionary algorithms (MOEAs) have been proposed in the literature. The earlier MOEAs such as VEGA [9], MOGA [10], and NSGA [11] have been followed by the elitist versions, e.g., NSGA-II [12] and SPEA2 [13]. More recent work has focused on the possible performance improvements by incorporating sophisticated strategies into MOEAs. For example, Jensen has employed advanced data structures to improve the runtime complexity of some popular MOEAs (e.g., NSGA-II) [14], while Yen et al. have proposed an approach based on the usage of dynamic populations [15]. In another recent work [16], the idea of transforming a high-dimentional multiobjective problem into a biobjective optimization problem is exploited within an MOEA.

In this paper, we extend the work of [8] with the following new contributions.

- We employ two state-of-the-art MOEAs [12], [13] in two case studies from SoC design and report performance results. Previously, these MOEAs have mostly been tested on simple and well-known mathematical functions, but detailed performance results on real-life engineering problems from different domains are very rare if any.

- A mathematical model is developed to exploit the multiprocessor mapping problem under multiple objectives.

- In order to determine the accuracy of the MOEAs, the mathematical model is first linearized and then solved by using an exact method, namely, the lexicographic weighted Chebyshev method.

- We perform two case studies in which we demonstrate: 1) the successful application of MOEAs to SoC design, especially in the early stages where the design space is very large; 2) the quantitative performance analysis of two state-of-the-art MOEAs examined in conjunction with an exact approach with respect to multiple criteria (e.g., accuracy, coverage of design space); and 3) the verification of multiobjective optimization results by further investigating a number of tradable solutions by means of simulation.

- In addition, we perform comparative experiments on variation operators and report performance results for different crossover types and mutation usage. More specifically, we analyze the consequences of using one-point, two-point, and uniform crossover operators on MOEA convergence and exploration of the search space. Besides, we also show that mutation still remains as a vital operator in multiobjective search to achieve good exploration. Hence, the MOEAs stay in accordance with the standard EAs in this respect.

- We define three new metrics which will allow us to compare different aspects of MOEAs.

- We examine the performance consequences of using different fitness assignment schemes (finer-grained and computationally more expensive versus more coarse-grained and computationally less expensive) in MOEAs.

- We study the outcome of using three different repair algorithms in constraint handling and compare them with respect to multiple criteria such as convergence and coverage of search space.

The rest of this paper is organized as follows. Section II discusses related work. Problem and model definitions and constraint linearizations are described in Section III. Section IV consists of four parts discussing the preliminaries for multiobjective optimization, the lexicographic weighted Chebyshev method, the different attributes of multiobjective evolutionary algorithms and the repair algorithm, and the metrics for comparing nondominated sets. In Section V, two case studies are performed and comparative performance analysis of MOEAs are given, followed by some simulation results. The last section presents concluding remarks.

\section{RELATED WORK}

In the domain of embedded systems and hardware/software codesign, several studies have been performed for system-level synthesis [17]-[19] and platform configuration [20]-[23]. The former means the problem of optimally mapping a task-level specification onto a heterogeneous hardware/software architecture, while the latter includes tuning the platform architecture parameters and exploring its configuration space.

Blickle etal. [17] partition the synthesis probleminto two steps: the selection of the architecture (allocation), and the mapping of the algorithm onto the selected architecture in space (binding) and time (scheduling). In their framework, they only consider cost and speed of the architecture, power consumption is ignored. To cope with infeasibility, they use penalty functions which reduce the number of infeasible individuals to an acceptable degree. In [19], a similar synthesis approach is applied to evaluate the design tradeoffs in packet processor architectures. But additionally, this model includes a real-time calculus to reason about packet streams and their processing. 
In the MOGAC framework [18], starting from a task graph specification, the synthesis problem is solved for three objectives: cost, speed, and power consumption of the target architecture. To accomplish this, an adaptive genetic algorithm which can escape local minima is utilized. However, this framework lacks the management of possible infeasibility as it treats all nondominated solutions equally even if they violate hard constraints. No repair algorithm is used in any stage of the search process; the invalid individuals are just removed at the end of evolution.

In [20], the configuration space of a parameterized SoC architecture is optimized with respect to a certain application mapped onto that architecture. The exploration takes into account power/performance tradeoffs and takes advantage of parameter dependencies to guide the search. The configuration space is first clustered by means of a dependency graph, and each cluster is searched exhaustively for local Pareto-optimal solutions. In the second step, the clusters are merged iteratively until a single cluster remains. The Pareto-optimal configurations within this last cluster form the global Pareto-optimal solutions. In [21], the exploration framework of [20] is used in combination with a simulation framework. The simulation models of SoC components (e.g., processors, memories, interconnect busses) are used to capture dynamic information which is essential for the computation of power and performance metrics. More recently, Ascia et al. [22] have also applied a genetic algorithm to solve the same problem.

The work in [23] presents an exploration algorithm for parameterized memory architectures. The inputs to the exploration algorithm are timing and energy constraints obtained from the application tasks and the memory architecture specifications. The goal is to identify the system time/energy tradeoff, when each task data member is assigned a target memory component. Exact and heuristic algorithms are given for solving different instances of the problem. However, only one type of heuristic (based on a branch and bound algorithm) is used, and no comparison with other heuristics is given.

In the Sesame framework, we do not target the problem of system synthesis. Therefore, a schedule is not constructed at the end of the design process. Our aim is to develop a methodology which allows for evaluating a large design space and provides us with a number of approximated Pareto-optimal solutions. These solutions are then input to our simulation framework for further evaluation. After simulation, figures about system-level tradeoffs (e.g., utilization of components, data throughput, communication media contention) are provided to the designer. Thus, our goal is efficient design space exploration in terms of simulation. In addition, our framework also differs from the mentioned frameworks in the sense that it uses process networks for algorithm specification rather than task graphs.

Most of the aforementioned system-level synthesis/exploration and platform configuration frameworks have relied on evolutionary search techniques. Besides these studies, evolutionary algorithms are utilized at many abstraction levels of electronic systems design, such as in analog integrated circuit synthesis [24] and in the design of digital signal processing systems [25] and evolvable hardware [26].

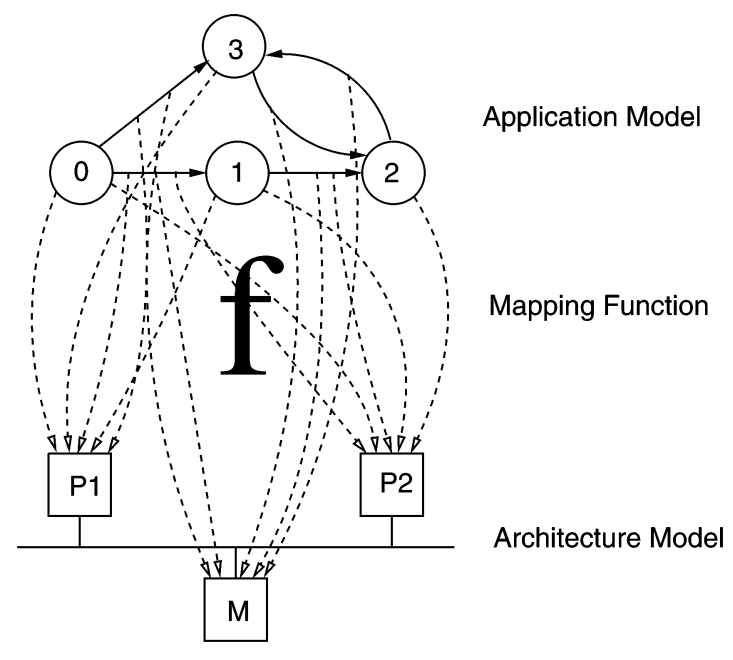

Fig. 1. The mapping problem on a simple example. The mapping function has to consider multiple conflicting design objectives and should identify the set of Pareto-optimal mappings.

\section{Problem AND Model Definition}

In the Sesame framework, applications are modeled using the Kahn process network (KPN) [27] model of computation in which parallel processes-implemented in a high-level language-communicate with each other via unbounded FIFO channels. The workload of an application is captured by instrumenting the code of each Kahn process with annotations. By executing the application model, each process generates its own trace of application events.

The architecture models in Sesame simulate the performance consequences of the application events generated by an application model. They solely account for performance constraints and only model timing behavior, since the functional behavior is already captured in the application model. An architecture model is constructed from generic building blocks provided by a library which contains template models for processing cores and various types of memory.

Since Sesame makes a distinction between application and architecture models, it needs an explicit mapping step to relate these models for cosimulation. In this step, the designer decides for each application process and first in, first out (FIFO) channel a destination architecture model component to simulate its workload. Thus, this step is one of the most important stages in the design process, since the final success of the design is highly dependent on these mapping choices. In Fig. 1, we illustrate this mapping step on a very simple example. In this example, the application model consists of four Kahn processes and five FIFO channels. The architecture model contains two processors and one shared memory. To decide on an optimum mapping, there exist multiple criteria to consider: maximum processing time in the system, power consumption, and the total cost. This section aims at defining a mapping function, shown with $\mathbf{f}$ in Fig. 1, to supply the designer with a set of best alternative mappings under the mentioned system criteria.

\section{A. Application Modeling}

The application models in Sesame are represented by a graph $\mathrm{KPN}=\left(V_{K}, E_{K}\right)$, where the set $V_{K}$ and $E_{K}$ refer to the Kahn 
nodes and the directed FIFO channels between these nodes, respectively. For each node $a \in V_{K}$, we define $B_{a} \subseteq E_{K}$ to be the set of FIFO channels connected to node $a, B_{a}=$ $\left\{b_{a 1}, \ldots, b_{a n}\right\}$. For each Kahn node, we define a computation requirement, shown with $\alpha_{a}$, representing the computational workload imposed by that Kahn node onto a particular component in the architecture model. The communication requirement of a Kahn node is not defined explicitly; rather it is derived from the channels attached to it. We have chosen this type of definition for the following reason: if the Kahn node and one of its channels are mapped onto the same architecture component, the communication overhead experienced by the Kahn node due to that specific channel is simply neglected. Only its channels that are mapped onto different architecture components are taken into account. So our model neglects internal communications and only considers external communications. Formally, we denote the communication requirement of the channel $b$ with $\beta_{b}$. To include memory latencies into our model, we require that mapping a channel onto a specific memory asks computation tasks from the memory. To express this, we define the computational requirement of the channel $b$ from the memory as $\alpha_{b}$. The formulation of our model ensures that the parameters $\beta_{b}$ and $\alpha_{b}$ are only taken into account when the channel $b$ is mapped onto an external memory.

\section{B. Architecture Modeling}

Similarly to the application model, the architecture model is also represented by a graph $A R C=\left(V_{A}, E_{A}\right)$, where the sets $V_{A}$ and $E_{A}$ denote the architecture components and the connections between the architecture components, respectively. In our model, the set of architecture components consists of two disjoint subsets: the set of processors $(P)$ and the set of memories $(M), V_{A}=P \cup M$ and $P \cap M=\emptyset$. For each processor $p \in P$, the set $M_{p}=\left\{m_{p 1}, \ldots, m_{p j}\right\}$ represents the memories which are reachable from the processor $p$. We define processing capacities for both the processors and the memories as $c_{p}$ and $c_{m}$, respectively. These parameters are set such that they reflect processing capabilities for processors, and memory access latencies for memories.

One of the key considerations in the design of embedded systems is the power consumption. In our model, we consider two types of power consumption for the processors. We represent the power dissipation of the processor $p$ during execution with $w_{p e}$, while $w_{p c}$ represents its power dissipation during communication with the external memories. For the memories, we only define $w_{m e}$, the power dissipation during execution. For both processors and memories, we neglect the power dissipation during idle times. In our model, we also consider the financial costs associated with the architecture model components. Using an architecture component in the system adds a fixed amount to the total cost. We represent the fixed costs as $u_{p}$ and $u_{m}$ for the processors and the memories, respectively.

\section{The Mapping Problem}

We have the following decision variables in the model: $x_{a p}=$ 1 if Kahn node $a$ is mapped onto processor $p, x_{b m}=1$ if channel $b$ is mapped onto memory $m, x_{b p}=1$ if channel $b$ is mapped onto processor $p, y_{p}=1$ if processor $p$ is used in the system, and $y_{m}=1$ if memory $m$ is used in the system. All the decision variables get a value of zero in all other cases. The constraints in the model are the following.

- Each Kahn node has to be mapped onto a single processor

$$
\sum_{p \in P} x_{a p}=1 \quad \forall a \in V_{K}
$$

- Each channel in the application model has to be mapped onto a processor or a memory

$$
\sum_{p \in P} x_{b p}+\sum_{m \in M} x_{b m}=1 \quad \forall b \in E_{K} .
$$

- If two communicating Kahn nodes are mapped onto the same processor, then the communication channel(s) between these nodes have to be mapped onto the same processor

$$
x_{a_{i} p} x_{a_{j} p}=x_{b p} \quad \forall b=\left(a_{i}, a_{j}\right) \in E_{K} .
$$

- The constraint given below ensures that when two communicating Kahn nodes are mapped onto two separate processors, the channel(s) between these nodes are to be mapped onto an external memory

$$
\begin{aligned}
x_{a_{i} p_{k}} x_{a_{j} p_{l}} \leq \sum_{m \in M_{p_{k}} \cap M_{p_{l}}} x_{b m} & \forall a_{i}, a_{j} \in V_{K}, \\
& \forall b \in B_{a_{i}} \cap B_{a_{j}}, \\
& \forall p_{k} \neq p_{l} \in P .
\end{aligned}
$$

- The following constraints are used to settle the values of $y_{p}$ and $y_{m} \mathrm{~s}$ in the model. We multiply the right-hand side of the first equation series by the total number of Kahn nodes and FIFO channels, since this gives an upper bound on the number of application model components that can be mapped to any processor. Similar logic is applied to the equations related with memory

$$
\begin{gathered}
\sum_{a \in V_{K}} x_{a p}+\sum_{b \in E_{K}} x_{b p} \leq\left(\left|V_{K}\right|+\left|E_{K}\right|\right) y_{p}, \quad \forall p \in P \\
\sum_{b \in E_{K}} x_{b m} \leq\left|E_{K}\right| y_{m} \quad \forall m \in M
\end{gathered}
$$

Three conflicting objective functions exist in the optimization problem.

- The first objective function tries to minimize the maximum processing time in the system. For each processor and memory, $f_{p}$ and $f_{m}$ represent the total processing time of the processor and memory, respectively. We also show the total time spent by the processor for the execution events as $f_{p}^{e}$ and for the communication events as $f_{p}^{c}$

$$
\begin{aligned}
f_{p} & =f_{p}^{e}+f_{p}^{c} \\
f_{p}^{e} & =\frac{1}{c_{p}} \sum_{a \in V_{K}} \alpha_{a} x_{a p} \\
f_{p}^{c} & =\frac{1}{c_{p}} \sum_{a \in V_{K}} x_{a p} \sum_{b \in B_{a}, m \in M_{p}} \beta_{b} x_{b m} \\
f_{m} & =\frac{1}{c_{m}} \sum_{b \in E_{K}} \alpha_{b} x_{b m} .
\end{aligned}
$$


So the objective function is expressed as

$$
\min \max _{i \in V_{A}} f_{i}
$$

- The second objective function tries to minimize the power consumption of the whole system. Similarly, $g_{p}$ and $g_{m}$ denote the total power consumption of processor $p$ and memory $m$

$$
\begin{aligned}
g_{p}= & f_{p}^{e} w_{p e}+f_{p}^{c} w_{p c} \\
g_{m}= & f_{m} w_{m e} \\
& \min \sum_{i \in V_{A}} g_{i} .
\end{aligned}
$$

- The last objective function aims at minimizing the total cost of the architecture model

$$
\min \sum_{p \in P} u_{p} y_{p}+\sum_{m \in M} u_{m} y_{m} .
$$

Definition 1 (MMPN problem): The multiprocessor mappings of process networks (MMPN) problem is the multiobjective integer optimization problem shown in (16) and (17) at the bottom of the page. For the sake of convenience, Table I presents the set of mathematical symbols for the MMPN problem.

\section{Constraint Linearizations}

In Section V, we will solve an instance of the MMPN problem using both exact and heuristic methods. Because the problem has some nonlinear constraints, one has to linearize them before using a mathematical optimizer. Next, we show how this is done.

Equation (3) can be linearized by replacing it with these three constraints

$$
\begin{aligned}
& x_{b p} \geq x_{a_{i} p}+x_{a_{j} p}-1 \\
& x_{b p} \leq x_{a_{i} p} \\
& x_{b p} \leq x_{a_{j} p} .
\end{aligned}
$$

Similarly, (4) can be linearized by introducing a new binary variable $x_{a_{i} p_{k} a_{j} p_{l}}=x_{a_{i} p_{k}} x_{a_{j} p_{l}}$ and adding the constraints

$$
\begin{aligned}
& x_{a_{i} p_{k} a_{j} p_{l}} \geq x_{a_{i} p_{k}}+x_{a_{j} p_{l}}-1 \\
& x_{a_{i} p_{k} a_{j} p_{l}} \leq x_{a_{i} p_{k}} \\
& x_{a_{i} p_{k} a_{j} p_{l}} \leq x_{a_{j} p_{l}} .
\end{aligned}
$$

Finally, (9) can be linearized by introducing the binary variable $x_{a p b m}=x_{a p} x_{b m}$ and adding the constraints

$$
\begin{aligned}
& x_{a p b m} \geq x_{a p}+x_{b m}-1 \\
& x_{a p b m} \leq x_{a p} \\
& x_{a p b m} \leq x_{b m} .
\end{aligned}
$$

TABLE I

TABLE OF SYMBOLS FOR THE MMPN PROBLEM

\begin{tabular}{ll}
\hline Application parameters \\
\hline$V_{K}$ & set of Kahn nodes \\
$E_{K}$ & set of channels \\
$B_{a}$ & set of channels connected to node $a$ \\
$\alpha_{a}$ & computation requirement of node $a$ \\
$\beta_{b}$ & communication requirement of channel $b$ \\
$\alpha_{b}$ & computation requirement of channel $b$ \\
\hline Architecture parameters \\
\hline$V_{A} \quad$ set of architecture components \\
$E_{A} \quad$ connectivity set of architecture components \\
$P$ & set of processors \\
$M$ & set of memories \\
$c_{p}$ & processing capacity of processor $p$ \\
$c_{m}$ & processing capacity of memory $m$ \\
$w_{p e}$ & power dissipation of $p$ during execution \\
$w_{p c}$ & power dissipation of $p$ during communication \\
$w_{m e}$ & power dissipation of $m$ during execution \\
$u_{p}$ & fixed cost of $p$ \\
$u_{m}$ & fixed cost of $m$ \\
\hline Binary decision variables \\
\hline$x_{a p}$ & whether $a$ is mapped onto $p$ \\
$x_{b m}$ & whether $b$ is mapped onto $m$ \\
$x_{b p}$ & whether $b$ is mapped onto $p$ \\
$y_{p}$ & whether $p$ is used \\
$y_{m}$ & whether $m$ is used \\
\hline Functions \\
\hline$f_{i}$ & total processing time of component $i$ \\
$g_{i}$ & total power dissipation of component $i$ \\
\hline & \\
\hline
\end{tabular}

\section{Multiobjective Optimization}

\section{A. Preliminaries}

Definition 2: A general multiobjective optimization problem with $k$ decision variables and $n$ objective functions is defined as

$$
\begin{aligned}
\operatorname{minimize} & \mathbf{f}(\mathbf{x})=\left(f_{1}(\mathbf{x}), \ldots, f_{n}(\mathbf{x})\right) \\
\text { subject to } & \mathbf{x} \in X_{f}
\end{aligned}
$$

where $\mathbf{x}$ represents a solution and $X_{f} \subseteq X$ is the set of feasible solutions. The objective function vector $\mathbf{f}(\mathbf{x})$ maps a decision vector $\mathbf{x}=\left(x_{1}, \ldots, x_{k}\right)$ in decision space $(X)$ to an objective vector $\mathbf{z}=\left(z_{1}, \ldots, z_{n}\right)$ in objective space $(Z)$.

Definition 3 (Dominance Relations): Given two objective vectors $\mathbf{z}^{1}$ and $\mathbf{z}^{2}$, we say the following.

- $\mathbf{z}^{1} \ll \mathbf{z}^{2}\left(\mathbf{z}^{1}\right.$ strictly dominates $\left.\mathbf{z}^{2}\right)$ iff $z_{i}^{1}<z_{i}^{2}, \forall i \in$ $\{1, \ldots, n\}$.

- $\mathbf{z}^{1}<\mathbf{z}^{2}\left(\mathbf{z}^{1}\right.$ dominates $\left.\mathbf{z}^{2}\right)$ iff $z_{i}^{1} \leq z_{i}^{2}$ and $\mathbf{z}^{\mathbf{1}} \neq \mathbf{z}^{\mathbf{2}}$, $\forall i \in\{1, \ldots, n\}$.

- $\mathbf{z}^{1} \leq \mathbf{z}^{2}\left(\mathbf{z}^{1}\right.$ weakly dominates $\left.\mathbf{z}^{2}\right)$ iff $z_{i}^{1} \leq z_{i}^{2}, \forall i \in$ $\{1, \ldots, n\}$.

- $\mathbf{z}^{1} \sim \mathbf{z}^{2}\left(\mathbf{z}^{1}\right.$ is incomparable with $\left.\mathbf{z}^{2}\right)$ iff $\exists i \neq j \in$ $\{1, \ldots, n\}$ such that $z_{i}^{1}<z_{i}^{2}$ and $z_{j}^{2}<z_{j}^{1}$.

$$
\begin{array}{ll}
\min & \mathbf{f}=\left(\max _{i \in V_{A}} f_{i}, \sum_{i \in V_{A}} g_{i}, \sum_{p \in P} u_{p} y_{p}+\sum_{m \in M} u_{m} y_{m}\right) \\
\text { s.t. } & (1),(2),(3),(4),(5),(6),(7),(8),(9),(10),(12),(13) \\
& x_{a p}, x_{b m}, x_{b p}, y_{p}, y_{m} \in\{0,1\} \quad \forall a \in V_{K}, \quad \forall b \in E_{K}, \\
& \forall m \in M, \quad \forall p \in P .
\end{array}
$$


Definition 4: A decision vector $\mathbf{x} \in A \subseteq X_{f}$ is said to be nondominated in set $A$ iff $\nexists \mathbf{a} \in A$ such that $\mathbf{f}(\mathbf{a})<\mathbf{f}(\mathbf{x})$.

Definition 5 (Nondominated Set and Front): The set containing only nondominated decision vectors $X_{n d} \subseteq X_{f}$ is called nondominated set. Its image on the objective space $Z_{n d}=\{\mathbf{z}$ : $\left.\mathbf{z}=\mathbf{f}(\mathbf{x}), \mathbf{x} \in X_{n d}\right\}$ is called nondominated front.

Definition 6 (Pareto Set and Front): The set $X_{\text {par }}=\{\mathbf{x}$ : $\mathbf{x}$ is nondominated in $\left.X_{f}\right\}$ is called Pareto set. Its image on the objective space $Z_{\text {eff }}=\left\{\mathbf{z}: \mathbf{z}=\mathbf{f}(\mathbf{x}), \mathbf{x} \in X_{\text {par }}\right\}$ is called Efficient set or equivalently Pareto front.

Definition 7 (Euclidean Distance): The Euclidean distance between two vectors (of dimension $n$ ) $\mathbf{z}^{1}$ and $\mathbf{z}^{2}$ is defined as $\left\|\mathbf{z}^{1}-\mathbf{z}^{2}\right\|=\sqrt{\sum_{i=1}^{n}\left(z_{i}^{1}-z_{i}^{2}\right)^{2}}$.

After these ground-laying definitions, in the rest of this section we first briefly explain an exact method for solving multiobjective optimization problems, namely the lexicographic weighted Chebyshev method which was introduced by Steuer and Choo [28]. Then, we will move to heuristic methods and introduce two state-of-the-art highly competitive MOEAs [12], [13]. The discussion on MOEAs is performed within the context of the MMPN problem, especially when it comes to those problem specific parameters. We conclude this section by defining three new metrics for MOEA performance comparisons.

\section{B. Lexicographic Weighted Chebyshev Method}

Definition 8 (Weakly Pareto-Optimal Point): A solution $\mathbf{x}^{*} \in X_{f}$ is weakly Pareto-optimal if there is no $\mathbf{x} \in X_{\text {par }}$ such that $\mathbf{f}(\mathbf{x}) \ll \mathbf{f}\left(\mathrm{x}^{*}\right)$.

The lexicographic weighted Chebyshev method [28] works in two steps. In the first step, we take a reference vector in objective space with components

$$
z_{i}^{\text {ref }}=\min \left\{f_{i}\left(\mathbf{x} \mid \mathbf{x} \in X_{f}\right)\right\}-\epsilon_{i}
$$

where $\epsilon_{i}$ are small positive values. Generally, it is common to set $\epsilon_{i}$ to the value which makes $z_{i}^{\text {ref }}=\left\lfloor\min \left\{f_{i}\left(\mathbf{x} \mid \mathbf{x} \in X_{f}\right)\right\}\right\rfloor$. In this step, we solve

$$
\begin{aligned}
\min & \alpha \\
\text { subject to } & \alpha \geq \lambda_{i}\left|f_{i}(\mathbf{x})-z_{i}^{\text {ref }}\right| \\
& \sum_{i=1}^{n} \lambda_{i}=1,0<\lambda_{i}<1 \text { and } \mathbf{x} \in X_{f}
\end{aligned}
$$

which guarantees weak Pareto optimality [29]. We denote the set of solutions found in this first step with $X_{w}$. In the second step, solutions in $X_{w}$ are checked for Pareto optimality using

$$
\min \sum_{\substack{i=1 \\ \mathbf{x} \in X_{w}}}^{n} f_{i}(\mathbf{x})
$$

and all weakly Pareto-optimal points are eliminated. After this step, the retained Pareto-optimal solutions form $X_{\text {par }}$.

In Fig. 2, we illustrate this graphically. The first step in the lexicographic weighted Chebyshev method can be considered as drawing probing rays emanating from $\mathbf{z}^{\text {ref }}$ toward the Pareto

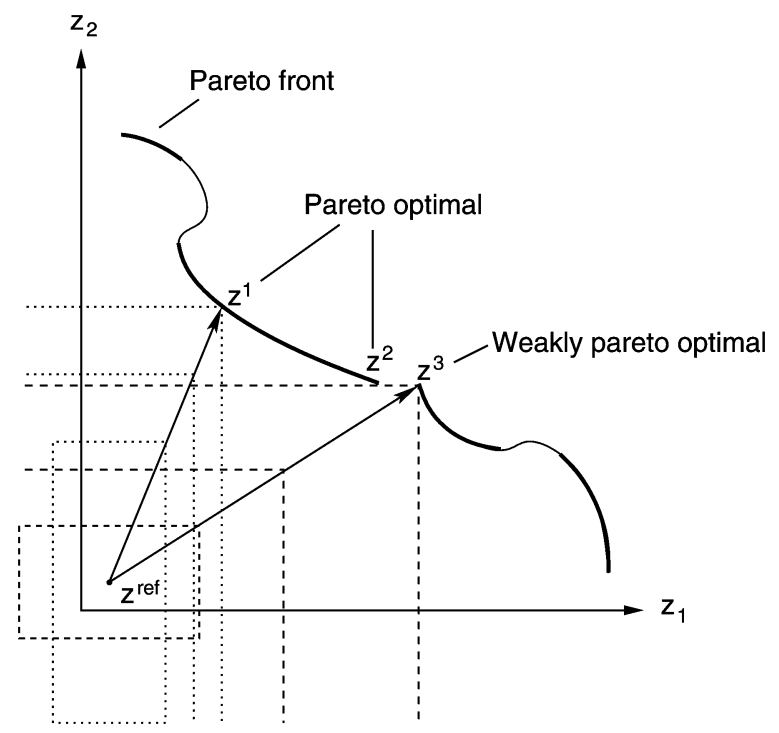

Fig. 2. The lexicographic weighted Chebyshev method can be considered as drawing probing rays emanating from $\mathbf{z}^{\text {ref }}$ toward the Pareto front. The points equidistant from $\mathbf{z}^{\text {ref }}$ form a family of rectangles centered at $\mathbf{z}^{\text {ref }}$.

front. The points equidistant from $\mathbf{z}^{\text {ref }}$ form a family of rectangles centered at $\mathrm{z}^{\text {ref }}$. Moreover, the vertices of these rectangles lie in the probing ray in the domain of the problem [30]. The objective in (27) is optimized when the probing ray intersects the Pareto front. In this step, points $\mathbf{z}^{1}, \mathbf{z}^{2}$ and $\mathbf{z}^{3}$ can be located. In the second step, weakly Pareto-optimal $\mathbf{z}^{3}$ is eliminated.

\section{Multiobjective Evolutionary Algorithms (MOEAs)}

Evolutionary algorithms have become very popular in multiobjective optimization, as they operate on a set of solutions. Over the years, many multiobjective evolutionary algorithms have been proposed [31], [32]. In this section, we study two state-of-the-art MOEAs: SPEA2, which was proposed by Zitzler et al. [13], and NSGA-II by Deb et al. [12]. Both algorithms are similar in the sense that they follow the main loop in Algorithm 1. To form the next generation, they employ a deterministic truncation by choosing $N$ best individuals from a pool of current and offspring populations. In addition, they both employ binary tournament selection [33]. Nevertheless, the main difference lies in their fitness assignment schemes. Despite the fact that both MOEAs apply a lexicographic fitness assignment scheme, objectives of which are to give first priority to nondominance and second priority to diversity, SPEA2 does so by using a finer-grained and therefore a more computationally expensive approach than its rival NSGA-II. The interesting question here is whether this additional computation effort pays off when we look at the overall performance of SPEA2 and NSGA-II. This issue is investigated experimentally in Section V.

Algorithm 1 A General Elitist Evolutionary Algorithm

$\begin{aligned} & \text { input: } N \text { : Size of the population } \\ & T: \text { Maximum number of generations. } \\ & \text { output: Nondominated individuals in } P_{t+1} .\end{aligned}$ 


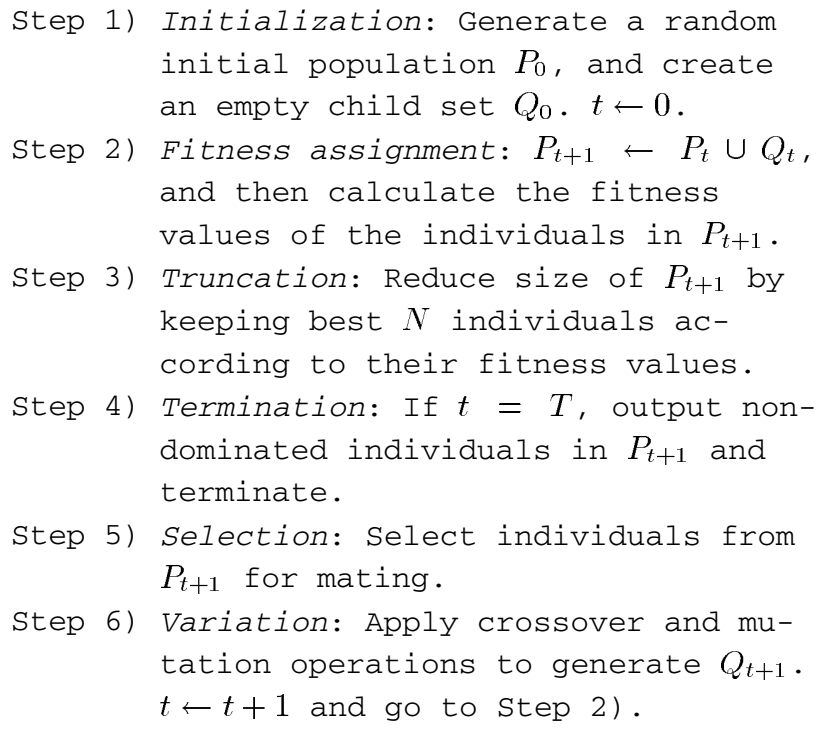

The distinctive characteristic of SPEA2 and NSGA-II is that both algorithms employ elitism, that is to guarantee a strictly positive probability for selecting at least one nondominated individual as an operand for variation operators. In both MOEAs, the following procedure is carried out to introduce elitism: the offspring and current population are combined and subsequently the best $N$ individuals in terms of nondominance and diversity are chosen to build the next generation. Unlike single optimization studies, elitism has attracted high attention from the researchers in multiobjective optimization. Although it is still a very active research subject, elitism is believed to be an important ingredient in search with multiple objectives. For example, in [34] and [35], experiments on continuous test functions show that elitism is beneficial, while in [36], similar results are also reported for two combinatorial (multiobjective 0/1 knapsack and traveling salesman) problems. Apart from these experimental studies, Rudolph has theoretically proven that an elitist MOEA can converge to the Pareto-front in finite number of iterations [37].

After the mentioned validatory studies, NSGA-II has been proposed as an elitist version of its predecessor NSGA. Besides elitism, NSGA-II has additional benefits over NSGA such as: 1) a lower computational complexity; 2) a parameterless mechanism for maintaining diversity among nondominated solutions; and 3) a deterministic selection algorithm to form the next generation by lexicographically sorting the combination of the current population and the offspring.

Similar to NSGA-II, SPEA2 is an improved successor of SPEA which was one of the first MOEAs with elitism. SPEA2 differs from SPEA in terms of: 1) a finer-grained fitness assignment mechanism; 2) a new density estimation technique for maintaining diversity; and 3) a new truncation method which prevents the loss of boundary solutions.

In the remainder of this section, we concentrate on problemspecific portions of MOEAs, and the discussion will be based on the MMPN problem, our focus of interest in this paper. The discussion is divided into three parts: individual encoding, constraint violations and variation operations. We conclude this sec-

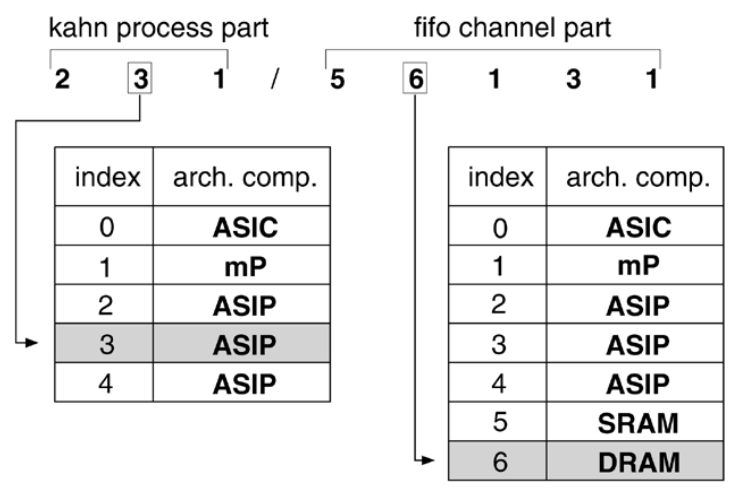

Fig. 3. An example individual coding. The closely related genes are put together in order to preserve locality.

tion by defining three new metrics in the interest of comparing MOEAs under different criteria.

1) Individual Encoding: Each genotype consists of two main parts: a part for Kahn process nodes and a part for FIFO channels. Each gene in the chromosome has its own feasible set which is determined by the type of the gene and the constraints of the problem. For genes representing Kahn process nodes, only the set of processors in the architecture model form the feasible set, while for genes representing the FIFO channels, both the set of processors and the set of memories constitute the feasible set.

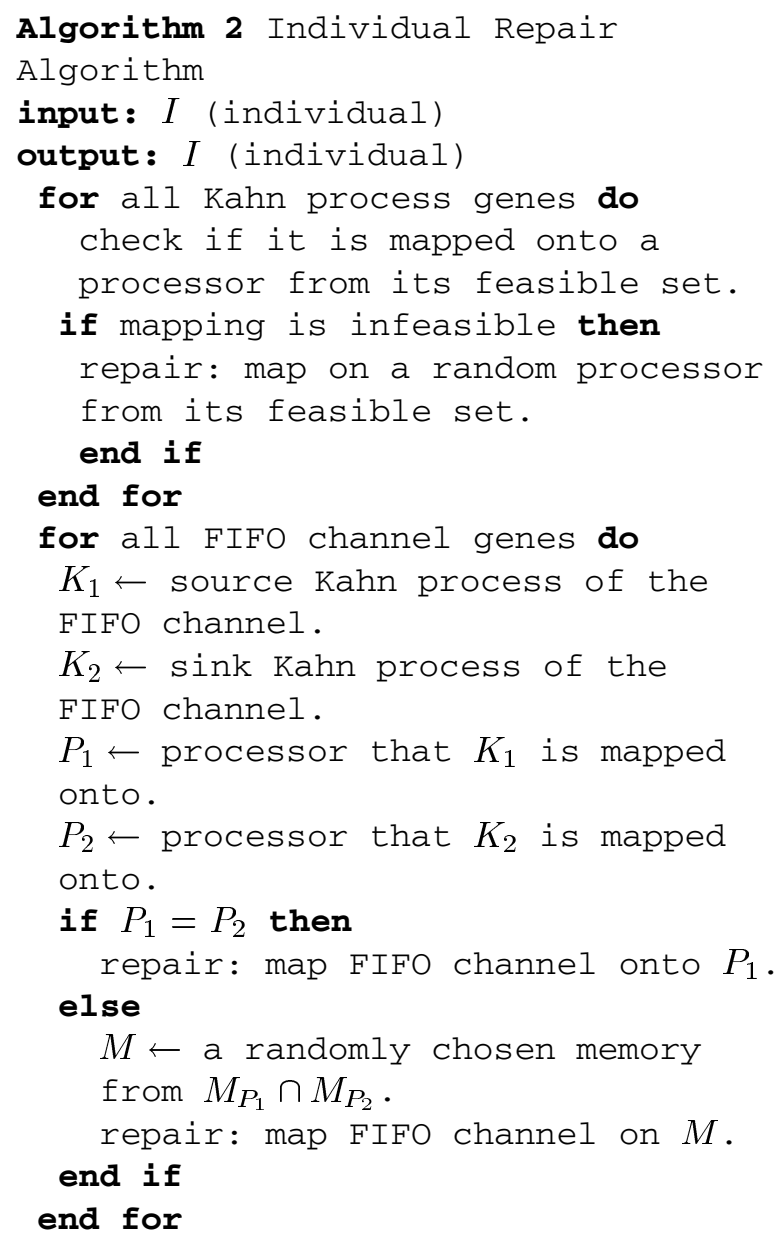


The constraints of the problem may include some limitations which should be considered in individual coding. For example, if there exists a dedicated architecture component for a specific Kahn process, then this architecture component has to be included only in the feasible set of this Kahn process.

In Fig. 3, an example chromosome is given. The first three genes are those for Kahn process nodes, and the rest are those for FIFO channels. We have placed closely related genes together in order to maintain locality. The latter is vital for the success of an evolutionary algorithm [18], [38]. For this gene, the second Kahn process is mapped onto an application specific instruction processor (ASIP) while the second FIFO channel is mapped onto a DRAM. We also see that the feasible sets for these two genes are different.

2) Constraint Violations: We have developed a repair mechanism to deal with constraint violations. Due to randomness in MOEAs (in initialization, crossover, and mutation steps), constraints (1), (2), (3), and (4) are prone to violation. The repair mechanism given in Algorithm 2 first considers whether each Kahn process is mapped onto a processor from its feasible set; if not, it repairs by randomly mapping the Kahn process to a feasible processor. After having finished processing the Kahn process genes, it proceeds along with the FIFO channel genes. For the latter, the repair algorithm simply checks for each FIFO channel whether the Kahn processes it is connected to are mapped onto the same processor. If this condition holds, then it ensures that the FIFO channel is also mapped onto that processor. If the condition does not hold, which means that the Kahn processes are mapped onto different processors (say, $P_{1}$ and $P_{2}$ ), it finds the set of memories reachable from both $P_{1}$ and $P_{2}$ (mathematically, $M_{P_{1}} \cap M_{P_{2}}$ ). Then, it selects a memory from this set randomly and maps the FIFO channel onto that memory. However, it is interesting to note here that if $M_{P_{1}} \cap$ $M_{P_{2}}=\emptyset$, then the problem itself may become infeasible. ${ }^{2}$ Therefore, we exclude these architectures.

With respect to repair, we have developed and tested three strategies. In the first (no-repair) strategy none of the individuals is repaired during any step; all are treated as valid individuals during the optimization process. Once the optimization is finished, repair is applied to the invalid individuals and all nondominated solutions are output. Although this approach does not sound very promising as it neglects infeasibility, it is included here for two reasons: the first reason is that some researchers have applied this strategy to multiobjective combinatorial problems and reported positive results [18]; the second reason is to see the performance gain/loss when constraint handling is taken into account. In the second strategy, which we call moderate-repair, at the end of each variation (Step 6 in Algorithm 1) all invalid individuals are repaired. This allows infeasible individuals to enter the mutation step. The latter may help to explore new feasible areas over unfeasible solutions. This is especially important for combinatorial problems in which the feasible region

\footnotetext{
${ }^{2}$ Although it is possible to repair by mapping both the FIFO channel and one of the Kahn processes onto the processor that the other Kahn process is mapped onto, this would require the individual to re-enter repair as it may cause additional infeasibilities for other FIFO channels. In the worst case, this can be an infinite loop.
}

may not be connected. The last strategy we employ here is called extensive-repair, as it repairs all invalid individuals immediately after every variation step. Hence, all individuals entering mutation are feasible. The experimental results concerning the repair strategies are discussed in Section V.

3) Variation Operations: As we have already mentioned, experiments in Section V should give us some feedback about i) whether the finer-grained computationally expensive fitness assignment in SPEA2 pays off and ii) the effect of using different repair schemes (no-repair, moderate-repair, and extensive-repair strategies). Therefore, we have fixed other factors that may effect MOEA performance. We have used only one type of mutation and crossover operations in all standard runs. For the former, we have used independent bit mutation (each bit of an individual is mutated independently with respect to bit mutation probability), while for the latter, standard one-point crossover (two parent chromosomes are cut at a random point and the sections after the cut point are swapped) is employed.

Many researchers have reported comparative performance results on different crossover types and mutation for traditional EAs solving single objective problems [38]-[40]. Therefore, it may well be interesting to perform similar comparative experiments with some variation operators in the multiobjective case. In this respect, we have performed additional experiments in Section V-B for the comparison of different crossover operators and the effect of mutation usage.

In our analysis with respect to crossover operators, we have compared the performance of the one-point crossover with that of the two-point and uniform crossover operators. In two-point crossover, the individual is considered as a ring formed by joining the ends together. The ring is cut at two random points forming two segments, and the two mating parents exchange one segment in order to create the children. One should note that the two-point crossover performs the same task as the one-point crossover by exchanging a single segment; however, it is more general. Uniform crossover is rather different from both one-point and two-point crossover; two parents are selected for reproducing two children, and for each bit position on the two children it is randomly decided which parent contributes its bit value to which child.

\section{Metrics for Comparing Nondominated Sets}

To properly evaluate and compare MOEA performances, one can identify three important criteria [41].

- Accuracy: The distance of the resulting nondominated set to the Pareto-optimal front should be minimal.

- Uniformity: The solutions should be well distributed (in most cases uniform).

- Extent: The nondominated solutions should cover a wide range for each objective function value.

Unlike single objective optimization problems, where the single aim is to locate a global optimum without being trapped at local optima, multiobjective optimization requires multiple aims to be satisfied simultaneously. Besides the obvious accuracy criterion, that is locating a set of solutions being at 


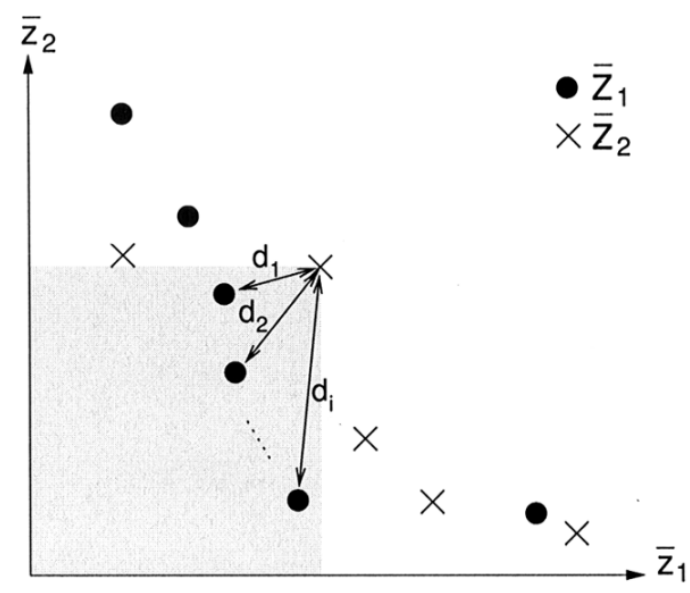

(a) D-metric for accuracy.

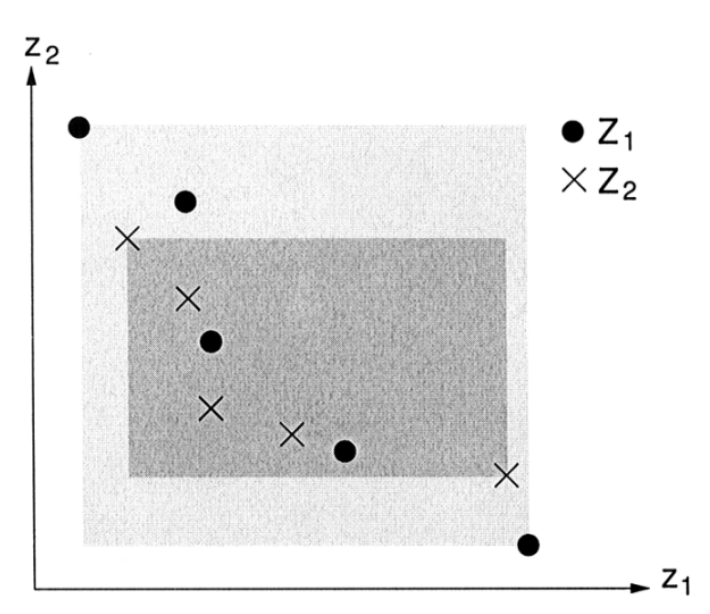

(c) $\nabla$-metric for extent.

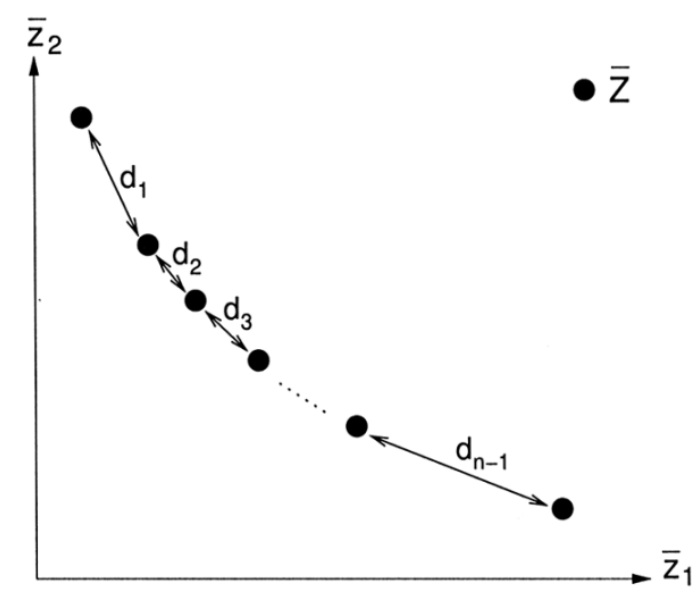

(b) $\Delta$-metric for uniformity.

\section{.}

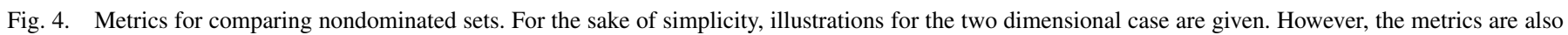
valid for any higher dimension.

minimal distant from the Pareto front, multiobjective optimizers also need to maintain a well distributed solution set (i.e., uniformity) for a more complete view of the tradeoff curve and should catch boundary points (i.e., extent) for a better coverage of the objective space.

There has been some effort for measuring the performance assessments of MOEAs [35], [42]. Metrics, in general, can be classified as: 1) metrics evaluating only one nondominated set; 2) metrics comparing two nondominated sets; 3) metrics requiring knowledge of the Pareto-optimal set; and 4) metrics measuring single or multiple assessment(s).

In the rest of this section, we propose one metric for each of the three identified criteria. Due to the fact that every objective function scales independently, one should map the limits of the objective function values to a unique interval before doing any arithmetic operation. Therefore, we first present normalization of vectors, before defining the performance metrics.

1) Normalization of Vectors in Objective Space: To make calculations scale independent, we normalize vectors before doing any arithmetic. At the end of normalization, each coordinate of the objective space is scaled such that all points get a value in the range $[0,1]$ for all objective values. Assume we have $p$ nondominated sets, $Z_{1}, \ldots, Z_{p}$. First, we form $Z=Z_{1} \cup \cdots \cup Z_{p}$. Then, we calculate $f_{i}^{\text {min }}=\min \left\{f_{i}(\mathbf{x}), \mathbf{f}(\mathbf{x})=\mathbf{z} \in Z\right\}$ and $f_{i}^{\max }=\max \left\{f_{i}(\mathbf{x}), \mathbf{f}(\mathbf{x})=\mathbf{z} \in Z\right\}$, which correspond to the minimum and maximum values for the $i$ th coordinate of the objective space. Then, we scale all points according to

$$
\bar{f}_{i}(\mathbf{x})=\frac{f_{i}(\mathbf{x})-f_{i}^{\min }}{f_{i}^{\max }-f_{i}^{\min }} .
$$

We repeat this process for all coordinates, i.e., $i=1 \ldots n$. We show the normalized vector of a vector $\mathbf{z}$ as $\overline{\mathbf{z}}$. Similarly, the set of normalized vectors are shown as $\bar{Z}$.

2) D-Metric for Accuracy: Given two normalized nondominated sets $\bar{Z}_{1}$ and $\bar{Z}_{2}, \forall \overline{\mathbf{a}} \in \bar{Z}_{1}$, we look for $\exists \overline{\mathbf{b}} \in \bar{Z}_{21} \subseteq \bar{Z}_{2}$ such that $\overline{\mathbf{b}}<\overline{\mathbf{a}}$. Then, we compute Euclidean distances from $\overline{\mathbf{a}}$ to all points $\overline{\mathbf{b}} \in \bar{Z}_{21}$. We define $\|\overline{\mathbf{a}}-\overline{\mathbf{b}}\|_{\max }=\max \{\| \overline{\mathbf{a}}-$ $\left.\overline{\mathbf{b}} \|, \overline{\mathbf{a}} \in \bar{Z}_{1}, \overline{\mathbf{b}} \in \bar{Z}_{21}\right\}$ to be the maximum of such distances. If $\bar{Z}_{21}=\emptyset$ then $\|\overline{\mathbf{a}}-\overline{\mathbf{b}}\|_{\max }=0$

$$
D\left(\bar{Z}_{1}, \bar{Z}_{2}\right)=\sum_{\overline{\mathbf{a}} \in \bar{Z}_{1}} \frac{\|\overline{\mathbf{a}}-\overline{\mathbf{b}}\|_{\max }}{\sqrt{n}\left|\bar{Z}_{1}\right|}
$$




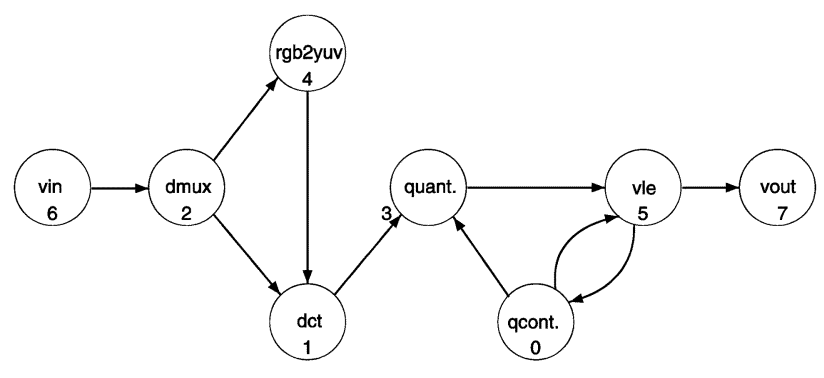

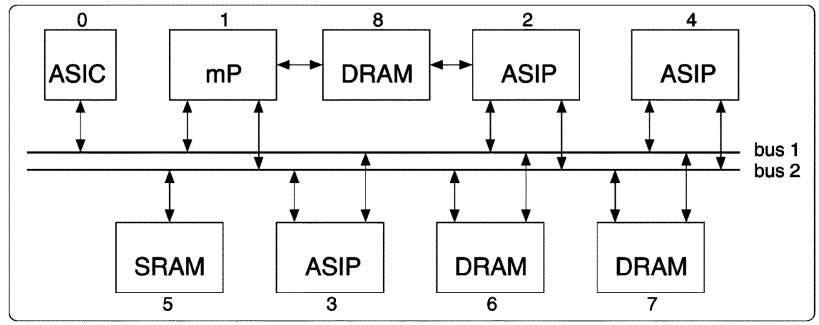

(a)

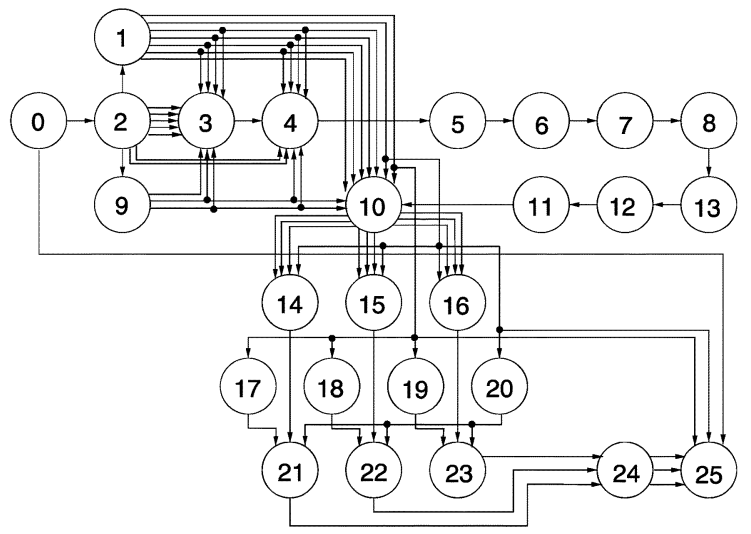

(b)

Fig. 5. Two multimedia applications and a platform architecture is shown. (a) M-JPEG encoder application and the multiprocessor SoC architecture model and (b) JPEG decoder process network with 26 processes and 75 FIFO channels.

where $n$ is the dimension of the objective space. The D-metric returns a value in the range $[0,1]$ where a smaller value is better. As seen in Fig. 4(a), the maximum distance from a dominating point is taken as a basis for accuracy.

3) $\Delta$-Metric for Uniformity: Given a normalized nondominated set $\bar{Z}$, we define $d_{i}$ to be the Euclidean distance between two consecutive vectors, $i=1 \ldots(|\bar{Z}|-1)$. Let $\hat{d}=$ $\sum_{i=1}^{|\bar{Z}|-1} d_{i} /|\bar{Z}|-1$. Then, we have

$$
\Delta(\bar{Z})=\sum_{i=1}^{|\bar{Z}|-1} \frac{\left|d_{i}-\hat{d}\right|}{\sqrt{n}(|\bar{Z}|-1)}
$$

where $n$ is the dimension of the objective space. Note that $0 \leq$ $\Delta(\bar{Z}) \leq 1$ where zero is the best. The underlying idea is to first calculate the average distance between any two consecutive points and then to check all distances and penalize with respect to the deviation from the average distance. In the ideal case, all distances $d_{1}, d_{2}, \ldots, d_{n-1}$ in Fig. 4(b) are equal to each other and the metric gets a value of zero.

4) $\nabla$-Metric for Extent: Given a nondominated set $Z$, we define $f_{i}^{\min }=\min \left\{f_{i}(\mathbf{x}), \mathbf{f}(\mathbf{x})=\mathbf{z} \in Z\right\}$ and $f_{i}^{\max }=$ $\max \left\{f_{i}(\mathbf{x}), \mathbf{f}(\mathbf{x})=\mathbf{z} \in Z\right\}$. Then

$$
\nabla(Z)=\prod_{i=1}^{n}\left|f_{i}^{\max }-f_{i}^{\min }\right|
$$

where $n$ is the dimension of the objective space. For this metric, normalization of vectors is not needed. As shown in Fig. 4(c), a bigger value spans a larger portion of the hypervolume and therefore is always better.

\section{EXPERIMENTS}

For the experiments, we have taken two media applications and a platform architecture to map the former onto the latter.

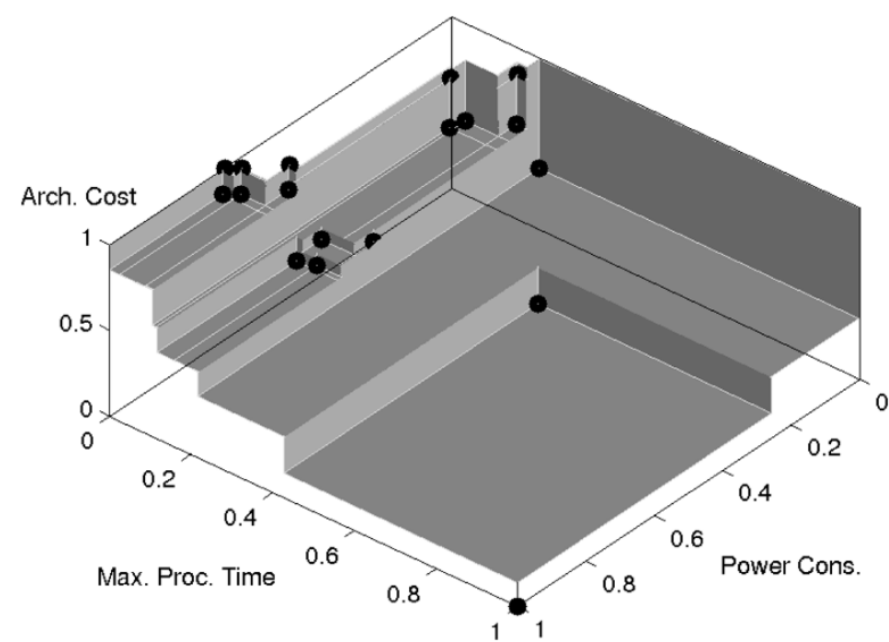

Fig. 6. Pareto front for the M-JPEG encoder case study.

The first application is a modified motion-JPEG encoder which differs from traditional encoders in three ways: it only supports lossy encoding while traditional encoders support both lossless and lossy encodings, it can operate on YUV and RGB video data whereas traditional encoders usually operate on the YUV format, and it can change quantization and Huffman tables dynamically while the traditional encoders have no such behavior. We omit giving further details on the M-JPEG encoder as they are not crucial for the experiments performed here. Interested readers are pointed to [43].

The second application is a Philips in-house JPEG decoder from [44]. Regarding this application, we only have the topology information but not the real implementation. Therefore, we have synthetically generated all its parameter values. Both media applications and the platform architecture are given in Fig. 5. Although these two applications match in terms of complexity, the JPEG 


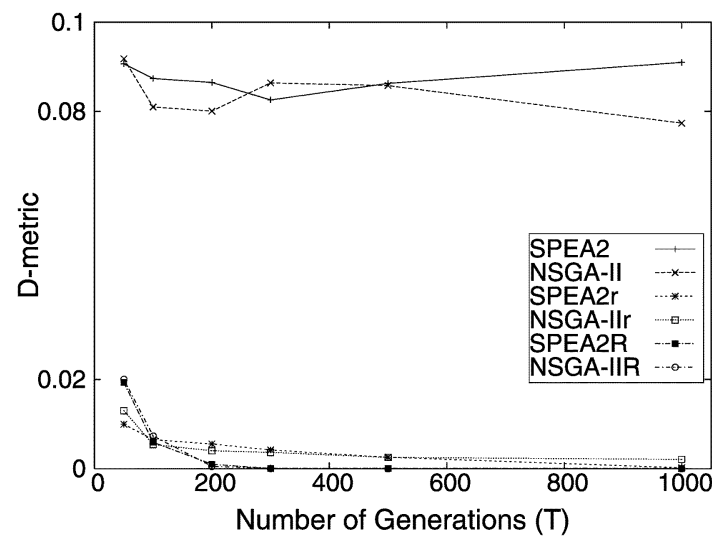

(a)

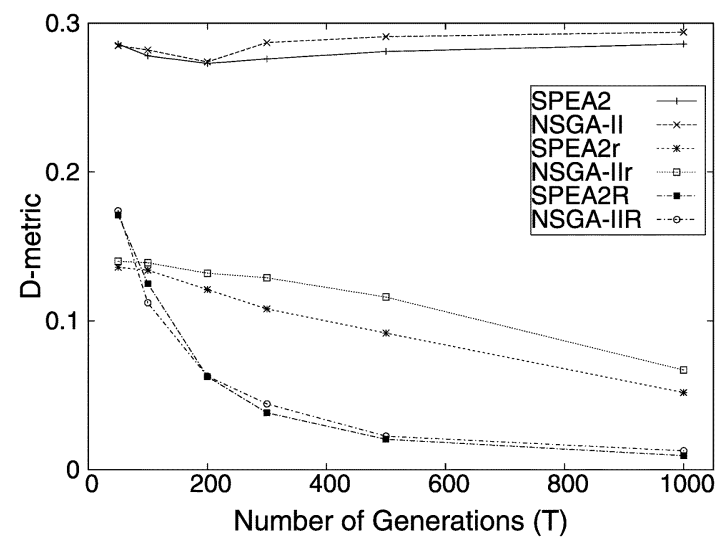

(b)

Fig. 7. Convergence analyses of the D-metric for the (a) M-JPEG encoder and (b) JPEG decoder. In the case of the M-JPEG decoder, the reference set is obtained by CPLEX, while for the JPEG decoder it is obtained by running SPEA2r with $T=10000$.

decoder has a more complex structure since its processes are defined at a finer granularity. In two case studies performed here, we have mapped these media applications successively onto the same platform architecture consisting of a general purpose microprocessor (mP), three ASIPs, an application-specific integrated circuit (ASIC), an SRAM, and three DRAMs. For these architecture components, realistic latency values from [43] have been used to calculate their processing capacities: $c_{p}$ and $c_{m}$. Similarly, for the Kahn processes and FIFO channels in the M-JPEG decoder, computational and communicational requirements (namely, the parameters $\alpha_{a}$ for the nodes and the parameters $\alpha_{b}$ and $\beta_{b}$ for the FIFO channels) have been calculated using statistics obtained from the $\mathrm{C}++$ implementation code of its Kahn process network.

We have implemented the MMPN problem as an optimization problem module in PISA-a platform and programming language-independent interface for search algorithms [45]. In PISA, the optimization process is split into two modules. One module contains all parts specific to the optimization problem such as individual encoding, fitness evaluation, mutation, and crossover. The other module contains the problem-independent parts such as selection and truncation. These two modules are implemented as two separate processes communicating through text files. The latter provides huge flexibility because a problem module can be freely combined with an optimizer module and vice versa. Due to the communication via file system, platform, programming language, and operating system independence are also achieved.

For M-JPEG encoder and JPEG decoder mapping problems, we have utilized the state-of-the-art highly competitive SPEA2 and NSGA-II multiobjective evolutionary optimizers. As already mentioned in Section IV-C2, we have used a repair algorithm (Algorithm 2) to handle constraint violations. In order to examine the effect of repair usage on the MOEA performance, we have utilized three different repair strategies (no-repair, moderaterepair, and intensive-repair), the details of which have already been discussed in Section IV-C2. In the rest of this paper, we present the results obtained under the no-repair strategy with SPEA2 (NSGA-II), while the results for the moderate-repair and intensive-repair strategies are shown by SPEA2r (NSGA-IIr) and SPEA2R (NSGA-IIR), respectively.
TABLE II

EXPERIMENTAL SETUP

\begin{tabular}{llll}
\hline MOEA & repair strategy & crossover & mutation \\
\hline SPEA2 & no-repair & one-point & indep. bit \\
SPEA2r & moderate-repair & one-point & indep. bit \\
SPEA2R & intensive-repair & one-point & indep. bit \\
NSGA-II & no-repair & one-point & indep. bit \\
NSGA-IIr & moderate-repair & one-point & indep. bit \\
NSGA-IIR & intensive-repair & one-point & indep. bit \\
\hline
\end{tabular}

In the experiments, we have used the standard one-point crossover and independent bit mutation variators. The population size is kept constant. All performance analyzes are carried out at different numbers of generations, ranging from 50 to 1000 , collecting information on the whole evolution of MOEA populations. The following values are used for the specific parameters:

- population size $N=100$;

- maximum number of generations

$$
T=50,100,200,300,500,1000 ;
$$

- mutation probability ${ }^{3} 0.5$, bit mutation probability ${ }^{4} 0.01$;

- crossover probability 0.8 .

The D-metric for measuring convergence to the Pareto front requires a reference set. To this end, we implemented the lexicographic weighted Chebyshev method and solved the M-JPEG encoder mapping problem by using the CPLEX mixed integer optimizer. ${ }^{5}$ The outcome of numerous runs with different weights has resulted in 18 Pareto-optimal points which are plotted in Fig. 6. The JPEG decoder is not solved by this exact

\footnotetext{
${ }^{3}$ The likelihood of mutating a particular solution.

${ }^{4}$ The likelihood of mutating each bit of a solution in mutation.

${ }^{5}$ ILOG CPLEX, http://www.ilog.com/products/cplex.
} 


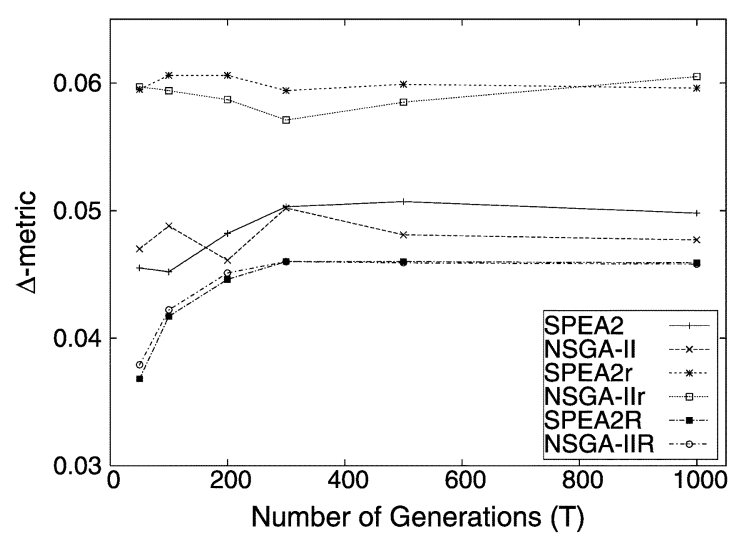

(a)

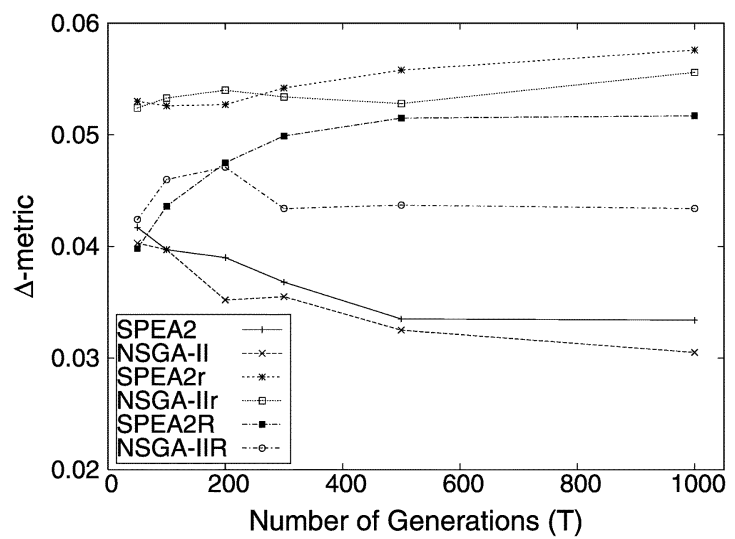

((b)

Fig. 8. Convergence analyses of the $\Delta$-metric for the (a) M-JPEG encoder and (b) JPEG decoder.

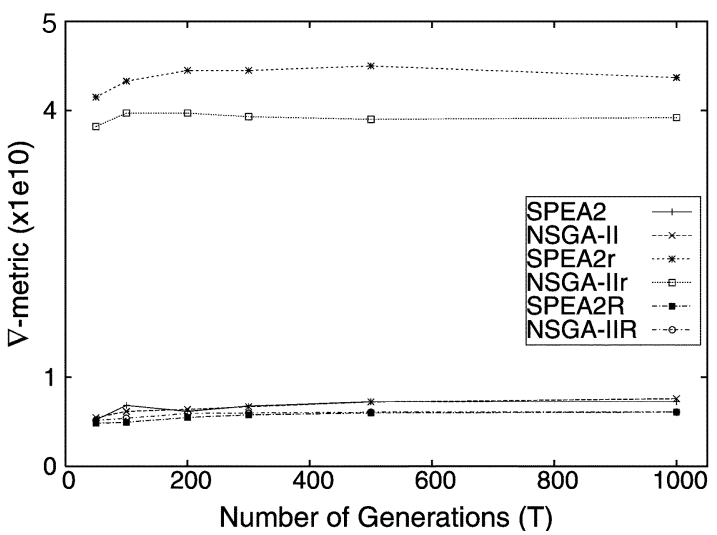

(a)

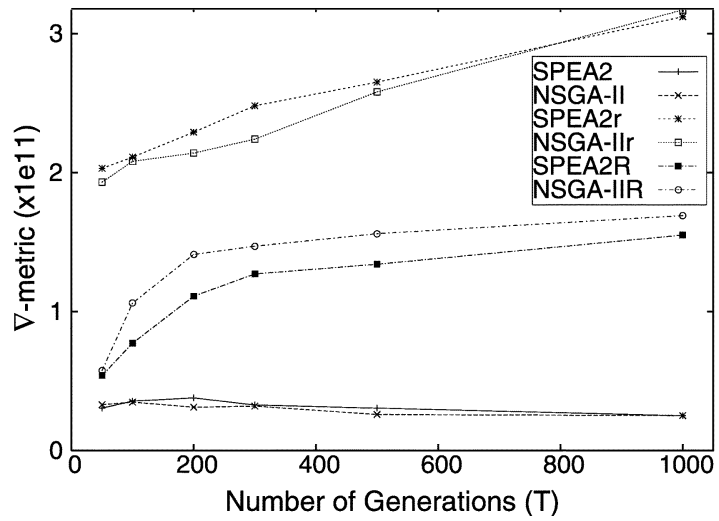

((b)

Fig. 9. Convergence analyses of the $\nabla$-metric for the (a) M-JPEG encoder and (b) JPEG decoder.

method due to its size; instead the result obtained by running SPEA2 for $T=10,000$ is taken as the reference set.

Table II summarizes the experimental setup. In the experiments, we have performed 30 runs (varying the random generator seed from one to 30) for each setup. An MOEA with some chosen $T$ makes up a setup: SPEA2 with $T=50$ or NSGA-IIR with $T=500$ are two examples. As a result, we have obtained 30 nondominated sets for each setup. All experiments have been performed on an Intel Pentium M PC with $1.7 \mathrm{GHz}$ CPU and 512 MB RAM running Linux OS.

\section{A. MOEA Performance Comparisons}

Table IV in the Appendix presents averages and standard deviations of the three performance metrics for each experimental setup with respect to 30 runs. The results for the same number of generations are grouped and compared. The best values obtained for all metrics are given in bold. To visualize the metrics convergence, we have plotted average metrics values against the numbers of generations in Figs. 7-9. We have the following conclusions.

- In terms of all three metrics, SPEA2 and NSGA-II score very close numbers and overall can be considered evenly matched. The same is true between SPEA2r and NSGA-IIr and also for SPEA2R and NSGA-IIR. However, with respect to run-times, NSGA-II, NSGA-IIr and

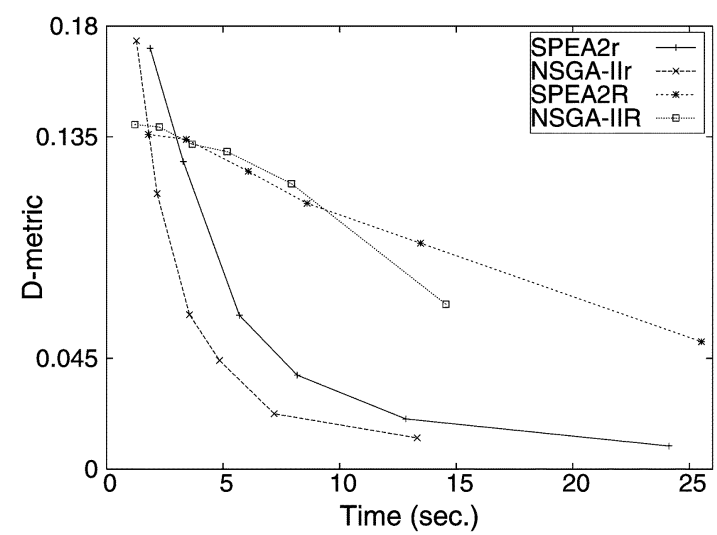

Fig. 10. Convergence with respect to time.

NSGA-IIR outperform SPEA2, SPEA2r and SPEA2R by only demanding on average $44 \%$ of their rivals' run-times. The latter is also demonstrated in Fig. 10, where we plot D-metric values with respect to wall clock time. Therefore, the finer-grained computationally expensive fitness assignment in SPEA2 (also in SPEA2r and SPEA2R) does not seem to pay off in general.

- In terms of accuracy (D-metric), SPEA2R and NSGA-IIR clearly outperform SPEA2r and NSGA-IIr. The worst performance is obtained when no repair is used, as clearly observed in Fig. 7. SPEA2 and NSGA-II fail to converge to 

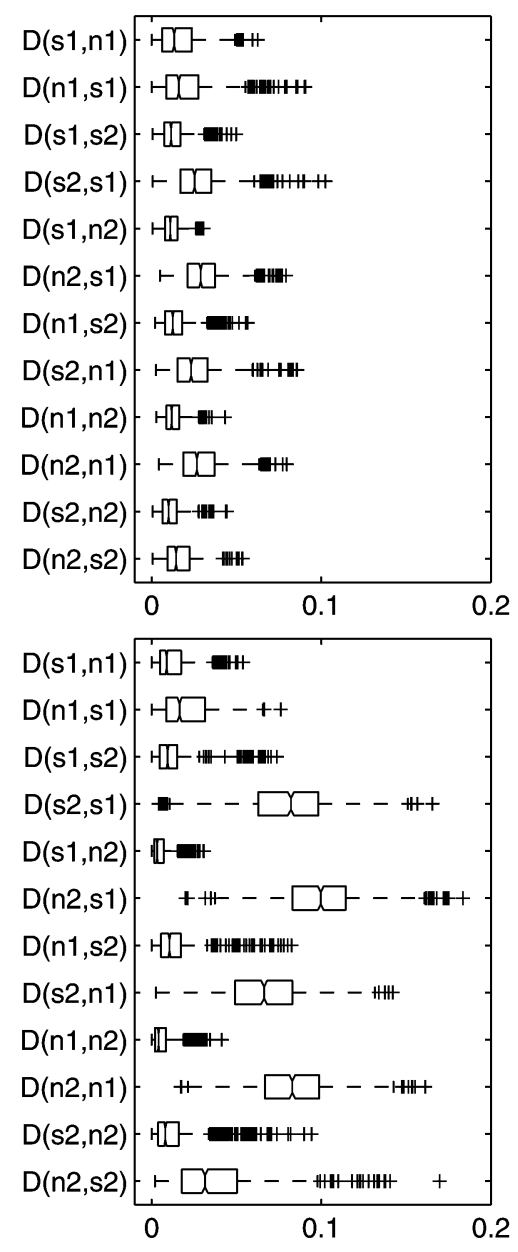
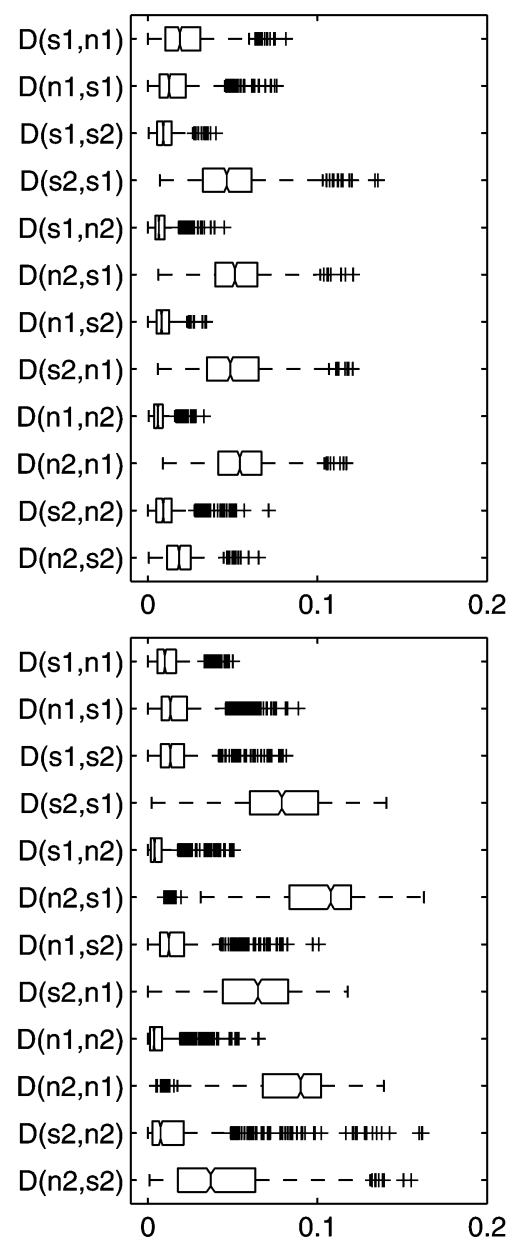
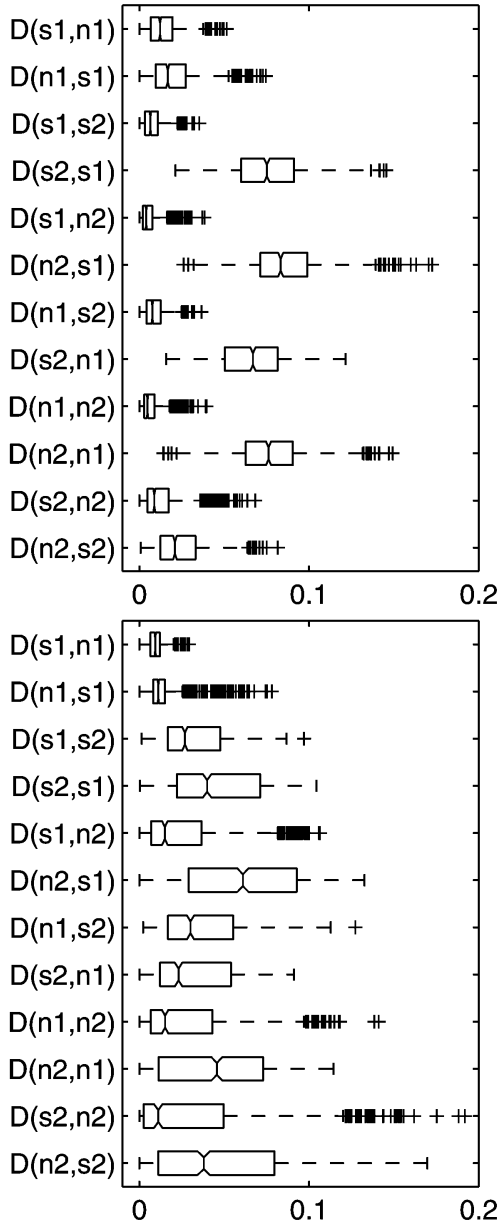

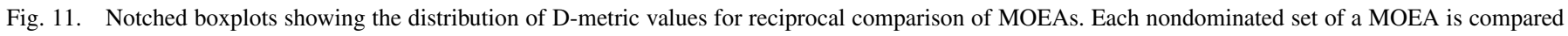

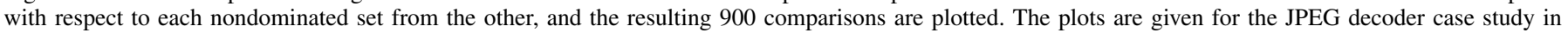

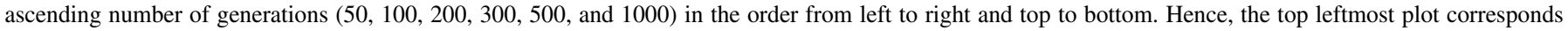

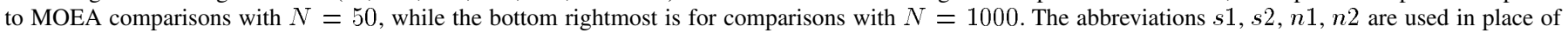

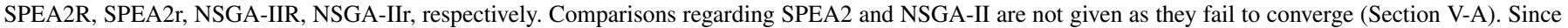
D-metric is nonsymmetric, i.e., $D\left(Z_{1}, Z_{2}\right) \neq D\left(Z_{2}, Z_{1}\right)$, both comparisons are performed. Note that smaller values are always better.vsk

the Pareto front. Therefore, constraint handling is of crucial importance.

- From D-metric plots in Figs. 7 and 10, we observe that convergence to the Pareto front accelerates with higher usage of repair. In this respect, we observe an exponential convergence curve for SPEA2R and NSGA-IIR, while the convergence of SPEA2r and NSGA-IIr approximates a linear curve. However, as the number of generations increase, the difference in terms of D-metric between SPEA2R (NSGA-IIR) and SPEA2r (NSGA-IIr) diminishes.

- In terms of uniformity, all algorithms perform indifferently. Although they start from a good initial value, they all fail to converge toward the optimal value zero. It is also very difficult to come to any conclusion about their behaviors from Fig. 8, e.g., it is unclear whether repair has any positive or negative effect on the $\Delta$-metric. Overall, all algorithms can be considered as good in terms of uniformity, as they all score below 0.06 , which is reasonably close to the optimal value.
- SPEA2r and NSGA-IIr clearly outperform other variants in terms of the extent metric. The reason behind this may be the higher explorative capacity of SPEA2r and NSGAIIr, as they can locate diverse feasible regions by mutating the invalid individuals. In this metric, SPEA2R and NSGA-IIR come second. Also from the $\nabla$-metric plot for JPEG decoder problem in Fig. 9, we observe convergence behavior for SPEA2r, NSGA-IIr, SPEA2R, NSGA-IIR, but not for SPEA2 and NSGA-II. Therefore, repair is essential for good extension but there seems to be no linear relation between the two.

- In the M-JPEG encoder case study, we compare nondominated sets generated by the MOEAs against the exact Pareto set (obtained by the lexicographic weighted Chebyshev method in CPLEX). As the numbers are very close to the ideal value zero for $T \geq 300$, especially for SPEA2R and NSGA-IIR, we consider them as highly promising optimizers. Convergence to Pareto front is also achieved with SPEA2r and NSGA-IIr, but this takes considerably larger amount of time. 

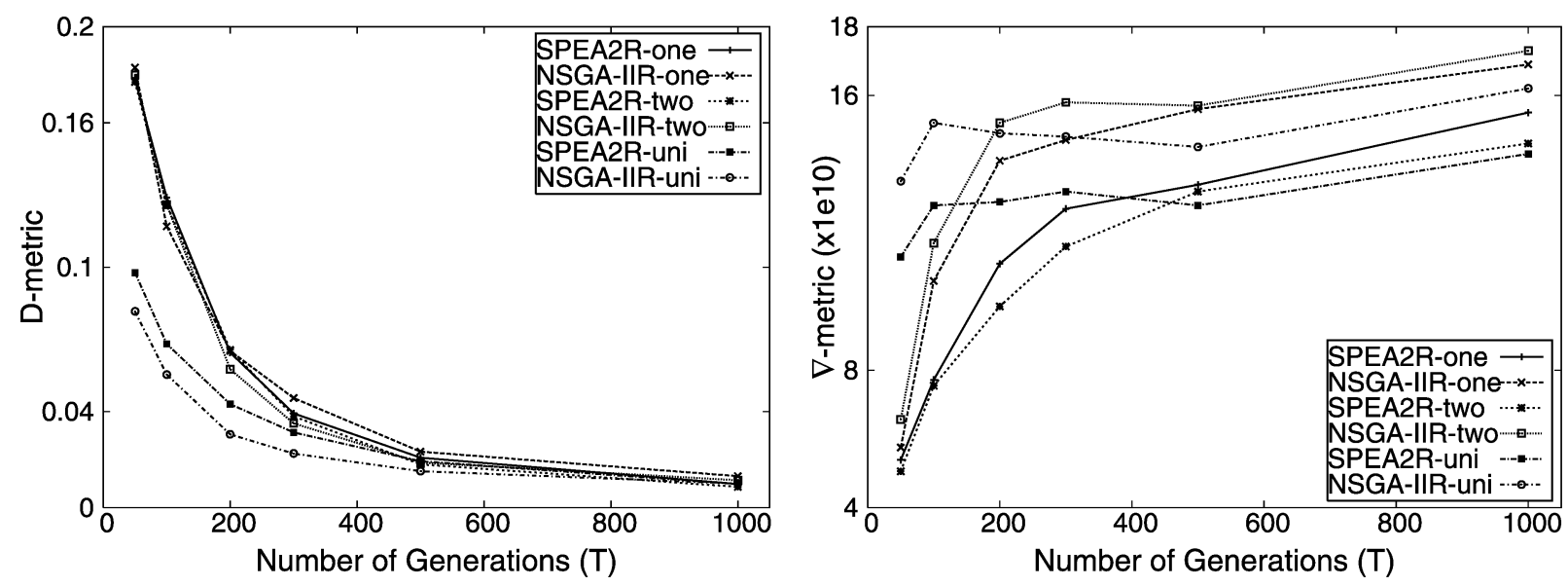

Fig. 12. Crossover analysis for the JPEG encoder case study.
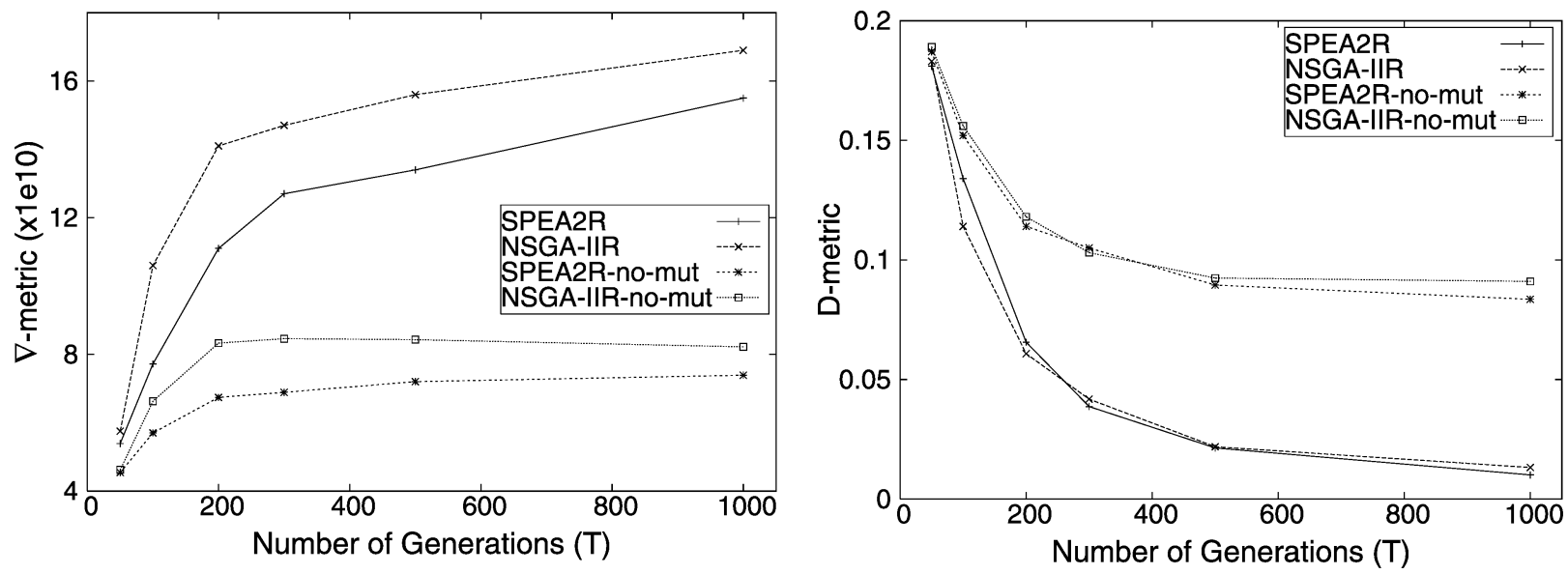

Fig. 13. Using no mutation results in poor $\nabla$-metric values in the JPEG encoder case study. This is an expected result as the mutation operator is responsible for exploration of the search space.

- In Fig. 11, we perform reciprocal comparison of nondominated sets at numbers of generations 50, 100, 200, 300, 500 , and 1000 for the JPEG decoder case study. To perform one comparison at a certain number of generations, 30 nondominated sets from an MOEA is compared one by one with the 30 nondominated sets from the other. The resulting distribution of $900 \mathrm{D}$-metric comparisons is given as a single notched boxplot in Fig. 11. The comparisons unveil that SPEA2R and NSGA-IIR beat SPEA2r and NSGA-IIr in all comparisons; and SPEA2R and NSGA-IIR can be considered as equally matched. The same is true between SPEA2r and NSGA-IIr. However, as the number of generations increase, the difference in D-metric between SPEA2R (NSGA-IIR) and SPEA2r (NSGA-IIr) diminishes as a result of the belated convergence of SPEA2r and NSGA-IIr. This difference in convergence speed is also apparent in Fig. 7, where we observe an exponential convergence curve for SPEA2R and NSGA-IIR in contrast to a linear curve for SPEA2r and NSGA-IIr.

\section{B. Effect of Crossover and Mutation}

In this section, we have performed two independent experiments with the JPEG decoder case study in order to analyze the effect of crossover and mutation operators on different MOEA performance criteria. The purpose of the first experiment is to examine the correlation between crossover type and convergence to the Pareto front. In this respect, besides the default one-point crossover operator, we have implemented two-point and uniform crossover operators (see Section IV-C3 for how they work). When we look at the resulting D-metric plots in Fig. 12, we observe a better performance with uniform crossover in the early generations; however after $T=500$, all crossover operators exhibit very close performance. With respect to extent ( $\nabla$-metrics in Fig. 12), two point crossover shows the worst performance, while once again one-point and uniform crossover operators match each other. The relatively fast convergence but coincidentally poor coverage of the search space in the case of the two-point crossover implies that the operator is biased more toward exploitation than exploration. One-point and uniform crossover operators seem to find a better balance of the two in this case.

In the second experiment, we analyze the effect of the mutation operator on MOEA performance. To realize this we have taken our original experimental setup (see Section V) and repeated the experiments without the mutation operator. The resulting D-metric and $\nabla$-metric plots are given in Fig. 13 . 
TABLE III

THREE SOLUTIONS CHOSEN FOR SIMULATION

\begin{tabular}{rccc}
\hline Solution & Max. Processing Time & Power Cons. & Arch. Cost \\
\hline cplex1 & 129338.0 & 1166.2 & 160.0 \\
cplex2 & 162203.7 & 966.9 & 130.0 \\
ad-hoc & 167567.3 & 1268.0 & 170.0 \\
\hline
\end{tabular}

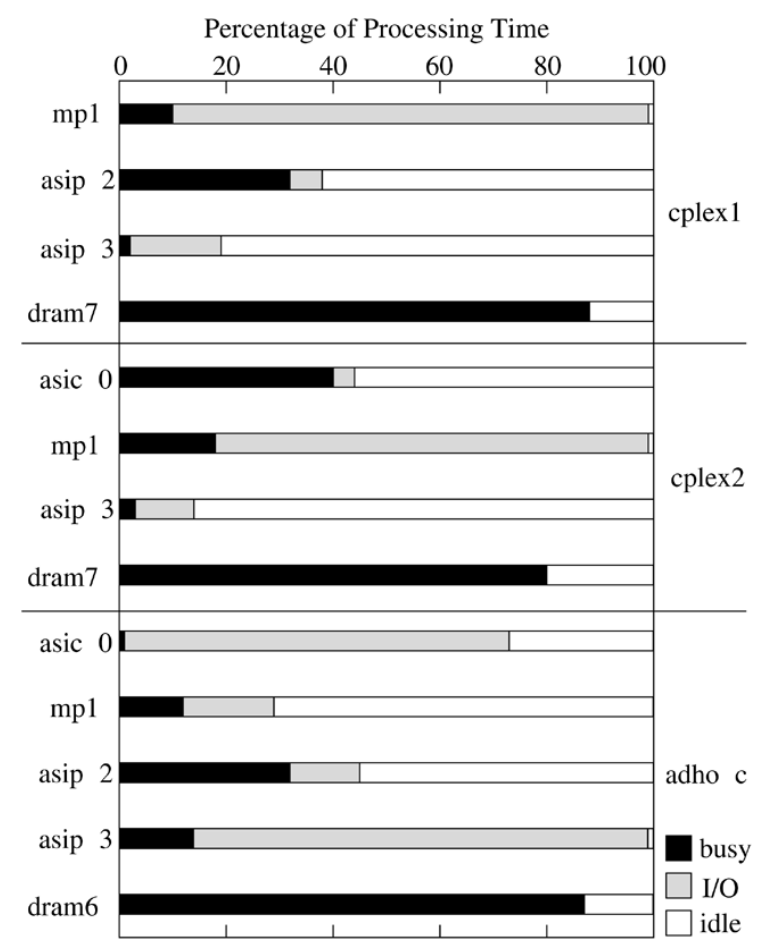

Fig. 14. Simulation results showing the utilization of architecture components in all three solutions. The throughput values are 52.2, 47.8, and 51.3 frames/s for the cplex 1 , cplex 2 and ad hoc solutions, respectively.

With respect to both metrics, omitting the mutation operator has resulted in very poor performance. MOEAs without mutation seem to converge toward the local optima and fail to collect variant solutions. Both observations imply that insufficient exploration has been realized in the search. These implications are in accordance with the widely accepted role of mutation as providing a reasonable level of population diversity in the standard EAs [39]. This experiment suggests that the explorative role of mutation is of high importance for MOEAs as well.

\section{Simulation Results}

In this section, we use the Sesame framework in order to evaluate three selected solutions of the M-JPEG encoder problem by means of simulation. Two of the solutions are taken from the Pareto-optimal set (referred here as cplex1 and cplex2), while the third solution is an ad hoc solution (referred as ad-hoc) which is very similar to those proposed and studied in [43] and [46]. It is clear from their objective function values in Table III that Pareto-optimal cplex 1 and cplex 2 outperform the ad-hoc solution in all objectives. The outcome of simulation experiments is also in accordance with optimization results, as the results in
Fig. 14 reveal that similar performance can be achieved using less processing cores (cplex 1 and cplex 2 use three while ad hoc uses four processors), which in turn results in less power consumption and cheaper implementation.

\section{CONCLUSION}

In this paper, we studied a multiobjective design problem from the multiprocessor system-on-chip domain: mapping process networks onto heterogeneous multiprocessor architectures. The mathematical model for the problem takes into account three objectives, namely, the maximum processing time, power consumption, and cost of the architecture, and is formulated as a nonlinear mixed integer programming. We have used an exact (lexicographic weighted Chebyshev) method and two state-of-the-art MOEAs (SPEA2 [13] and NSGA-II [12]) in order to locate the Pareto-optimal solutions. To apply the exact method, we first linearized the mathematical model by adding additional binary decision variables and new constraints.

Three new performance metrics have been defined to measure three attributes (accuracy, uniformity, and extent) of nondominated sets. These metrics are subsequently used to compare SPEA2 and NSGA-II with each other and also with the Pareto-optimal set. The two elitist MOEAs mainly differ in their fitness assignment schemes. SPEA2 uses a finer-grained and computationally more expensive scheme with respect to its rival NSGA-II. Performing two case studies, we have shown that SPEA2 is not superior than NSGA-II in any of the three defined metrics. Therefore, regarding the MMPN problem, the computationally more expensive fitness assignment scheme of SPEA2 does not seem to pay off, as NSGA-II is on average 2.2 times faster. Comparing the two MOEAs with the exact set in the M-JPEG encoder case study, we have shown that both SPEA2 and NSGA-II find solution sets very close to the Pareto-optimal set.

Constraint violations have been tackled by three repair strategies differing in terms of repair intensity, and the outcome of each strategy is evaluated with respect to the defined metrics. The main result is that using sufficient amount of repair is necessary for good convergence, but allowing some infeasibility may help the MOEA to explore new feasible regions over infeasible solutions. Thus, a balance should be established in terms of repair frequency.

Additionally, one-point, two-point, and uniform crossover operators have been comparatively evaluated in terms of accuracy and extent. To summarize, one-point and uniform crossover operators seem to find a good balance of exploitation vs. exploration, while two-point crossover is more biased toward exploitation. With respect to mutation usage, the experiments reveal that mutation retains its importance for exploration.

We have also compared and simulated two Pareto-optimal solutions and one ad hoc solution from previous studies [43], [46]. The results indicate that multiobjective search of the design space improves all three objectives, i.e., a cheaper implementation using less power but still performing the same in terms of system throughput can be achieved. 
TABLE IV

Performance Comparison of the MOEAs for the M-JPEG EnCoder AND JPEG DeCOder Applications. Best Values ARE IN Bold

\begin{tabular}{|c|c|c|c|c|c|c|c|c|c|c|c|c|c|}
\hline \multirow{3}{*}{ MOEA } & \multirow{3}{*}{$\mathrm{T}$} & \multicolumn{6}{|c|}{ M-JPEG encoder } & \multicolumn{6}{|c|}{ JPEG decoder } \\
\hline & & \multirow{2}{*}{\multicolumn{2}{|c|}{\begin{tabular}{l}
\multicolumn{2}{c}{ D-metric } \\
avg. $\quad$ std. dev.
\end{tabular}}} & \multicolumn{2}{|c|}{$\Delta$-metric } & \multicolumn{2}{|c|}{$\nabla$-metric } & \multicolumn{2}{|c|}{ D-metric } & \multicolumn{2}{|c|}{$\Delta$-metric } & \multicolumn{2}{|c|}{$\nabla$-metric } \\
\hline & & & & avg. & std. dev. & avg. & std. dev. & avg. & std. dev. & avg. & std. dev. & avg. & std. dev. \\
\hline SPEA2 & & $9.07 \mathrm{e}-2$ & $2.77 \mathrm{e}-2$ & $4.55 \mathrm{e}-2$ & $1.12 \mathrm{e}-2$ & $5.22 \mathrm{e} 9$ & $2.58 \mathrm{e} 9$ & $2.86 \mathrm{e}-1$ & $1.60 \mathrm{e}-2$ & $4.17 \mathrm{e}-2$ & $8.19 \mathrm{e}-3$ & $3.04 \mathrm{e} 10$ & $1.18 \mathrm{e} 10$ \\
\hline NSGA-II & & $9.18 \mathrm{e}-2$ & $2.25 \mathrm{e}-2$ & $4.70 \mathrm{e}-2$ & $1.35 \mathrm{e}-2$ & $5.47 \mathrm{e} 9$ & $3.01 \mathrm{e} 9$ & $2.85 \mathrm{e}-1$ & $2.18 \mathrm{e}-2$ & $4.03 e-2$ & $7.60 \mathrm{e}-3$ & $3.29 \mathrm{e} 10$ & $9.54 \mathrm{e} 9$ \\
\hline SPEA2r & 50 & $9.94 e-3$ & $8.93 e-3$ & $5.95 \mathrm{e}-2$ & $1.15 \mathrm{e}-2$ & 4.15e10 & $8.65 \mathrm{e} 9$ & $1.36 \mathrm{e}-1$ & $2.54 \mathrm{e}-2$ & $5.30 \mathrm{e}-2$ & $8.10 \mathrm{e}-3$ & $2.03 \mathrm{e} 11$ & $3.75 \mathrm{e} 10$ \\
\hline NSGA-IIr & 50 & $1.30 \mathrm{e}-2$ & $1.28 \mathrm{e}-2$ & $5.97 \mathrm{e}-2$ & $1.05 \mathrm{e}-2$ & $3.82 \mathrm{e} 10$ & $9.55 \mathrm{e} 9$ & $1.40 \mathrm{e}-1$ & $1.62 \mathrm{e}-2$ & $5.24 \mathrm{e}-2$ & $5.58 \mathrm{e}-3$ & $1.93 \mathrm{e} 11$ & $2.33 \mathrm{e} 10$ \\
\hline SPEA2R & & $1.93 \mathrm{e}-2$ & $2.27 \mathrm{e}-2$ & $3.68 \mathrm{e}-2$ & $7.27 e-3$ & $4.84 \mathrm{e} 9$ & $2.37 \mathrm{e} 9$ & $1.71 \mathrm{e}-1$ & $1.74 \mathrm{e}-2$ & 3.98e-2 & $5.12 \mathrm{e}-3$ & $5.39 \mathrm{e} 10$ & $1.41 \mathrm{e} 10$ \\
\hline NSGA-IIR & & $2.00 \mathrm{e}-2$ & $2.26 \mathrm{e}-2$ & $3.79 \mathrm{e}-2$ & $6.27 \mathrm{e}-3$ & $5.17 \mathrm{e} 9$ & $1.63 \mathrm{e} 9$ & $1.74 \mathrm{e}-1$ & $1.57 \mathrm{e}-2$ & $4.24 \mathrm{e}-2$ & $5.51 \mathrm{e}-3$ & $5.75 \mathrm{e} 10$ & $2.02 \mathrm{e} 10$ \\
\hline PEA2 & & $74 \mathrm{e}-2$ & $2.54 \mathrm{e}-2$ & $4.52 \mathrm{e}-2$ & $1.23 \mathrm{e}-2$ & $6.84 \mathrm{e} 9$ & 4.1. & $2.78 \mathrm{e}-1$ & $1.80 \mathrm{e}-2$ & $3.97 e-2$ & $7.47 e-3$ & $3.55 \mathrm{e} 10$ & $1.02 \mathrm{e} 10$ \\
\hline NSGA-II & & $10 \mathrm{e}-2$ & $1.81 \mathrm{e}-2$ & $4.88 \mathrm{e}-2$ & $9.81 \mathrm{e}-3$ & $6.17 \mathrm{e} 9$ & $2.17 \mathrm{e} 9$ & $2.82 \mathrm{e}-1$ & $1.87 \mathrm{e}-2$ & $3.97 \mathrm{e}-2$ & $7.83 e-3$ & $.48 \mathrm{e} 10$ & $1.16 \mathrm{e} 10$ \\
\hline SPEA2r & & $6.50 \mathrm{e}-3$ & $1.01 \mathrm{e}-2$ & $6.06 \mathrm{e}-2$ & $1.19 \mathrm{e}-2$ & $4.33 \mathrm{e} 10$ & $9.71 \mathrm{e} 9$ & $1.34 \mathrm{e}-1$ & $2.47 \mathrm{e}-2$ & $5.26 \mathrm{e}-2$ & $6.95 e-3$ & 2.11e11 & $4.00 \mathrm{e} 10$ \\
\hline NSGA-IIr & 100 & $5.41 \mathrm{e}-3$ & $8.41 \mathrm{e}-3$ & $5.94 \mathrm{e}-2$ & $8.44 \mathrm{e}-3$ & $3.97 \mathrm{e} 10$ & $8.59 \mathrm{e} 9$ & $1.39 \mathrm{e}-1$ & $1.94 \mathrm{e}-2$ & $5.33 \mathrm{e}-2$ & $5.51 \mathrm{e}-3$ & $2.08 \mathrm{e} 11$ & $3.64 \mathrm{e} 10$ \\
\hline SPEA2R & & $5.90 e-3$ & $1.15 \mathrm{e}-2$ & $4.17 \mathrm{e}-2$ & $6.30 \mathrm{e}-3$ & $4.95 \mathrm{e} 9$ & 1.7 & $1.25 \mathrm{e}-1$ & $2.02 \mathrm{e}-2$ & $4.36 \mathrm{e}-2$ & $4.53 e-3$ & $7.72 \mathrm{e} 10$ & $2.09 \mathrm{e} 10$ \\
\hline NSGA-IIR & & $7.32 \mathrm{e}-3$ & $1.64 \mathrm{e}-2$ & $4.22 \mathrm{e}-2$ & $5.26 \mathrm{e}-3$ & $5.40 \mathrm{e} 9$ & $1.56 \mathrm{e} 9$ & $1.12 \mathrm{e}-1$ & $2.20 \mathrm{e}-2$ & $4.60 \mathrm{e}-2$ & $4.68 \mathrm{e}-3$ & $1.06 \mathrm{e} 11$ & $3.25 \mathrm{e} 10$ \\
\hline PEA2 & & $8.65 \mathrm{e}-2$ & $2.12 \mathrm{e}-2$ & $4.82 \mathrm{e}-2$ & & & & $2.73 e-1$ & $2.27 \mathrm{e}-2$ & $3.90 \mathrm{e}-2$ & $8.15 e-3$ & $3.78 \mathrm{e} 10$ & $1.44 \mathrm{e} 10$ \\
\hline NSGA-II & & $8.01 \mathrm{e}-2$ & $1.68 \mathrm{e}-2$ & $4.61 \mathrm{e}-2$ & $1.16 \mathrm{e}-2$ & $6.40 \mathrm{e} 9$ & & $2.74 \mathrm{e}-1$ & $2.18 \mathrm{e}-3$ & 3.52e-2 & $6.18 \mathrm{e}-3$ & $1 \mathrm{e} 10$ & $9.54 \mathrm{e} 9$ \\
\hline PEA2r & & $5.55 e-3$ & $1.06 \mathrm{e}-2$ & $6.06 \mathrm{e}-2$ & $1.16 \mathrm{e}-2$ & $4.45 \mathrm{e} 10$ & $9.57 \mathrm{e} 9$ & $1.21 \mathrm{e}-1$ & $2.30 \mathrm{e}-2$ & $5.27 \mathrm{e}-2$ & $7.18 \mathrm{e}-3$ & $2.29 \mathrm{e} 11$ & $4.38 \mathrm{e} 10$ \\
\hline NSGA-IIr & & $4.01 \mathrm{e}-3$ & $8.00 \mathrm{e}-3$ & $5.87 \mathrm{e}-2$ & $8.68 \mathrm{e}-3$ & $3.97 \mathrm{e} 10$ & $9.61 \mathrm{e} 9$ & $1.32 \mathrm{e}-1$ & $2.31 \mathrm{e}-2$ & $5.40 \mathrm{e}-2$ & $5.92 \mathrm{e}-3$ & $2.14 \mathrm{e} 11$ & $4.02 \mathrm{e} 10$ \\
\hline SPEA2R & & $9.85 e-4$ & $2.29 \mathrm{e}-3$ & 4.46e-2 & $3.45 \mathrm{e}-3$ & $5.48 \mathrm{e} 9$ & $1.49 \mathrm{e} 9$ & $6.25 \mathrm{e}-2$ & $1.79 \mathrm{e}-2$ & $4.75 \mathrm{e}-2$ & $5.23 e-3$ & $1.11 \mathrm{e} 11$ & $2.91 \mathrm{e} 10$ \\
\hline NSGA-IIR & & $5.50 \mathrm{e}-4$ & $2.08 \mathrm{e}-3$ & $4.51 \mathrm{e}-2$ & $3.30 \mathrm{e}-3$ & $5.94 \mathrm{e} 9$ & $8.62 \mathrm{e} 8$ & $6.28 \mathrm{e}-2$ & $1.71 \mathrm{e}-2$ & $4.71 \mathrm{e}-2$ & $4.03 e-3$ & $1.41 \mathrm{e} 11$ & $3.57 \mathrm{e} 10$ \\
\hline Let & & $6 e-2$ & $1.98 \mathrm{e}-2$ & $33 e-2$ & & & & & & & & & $1.15 \mathrm{e} 10$ \\
\hline NSGA-II & & $64 e-2$ & $2.15 \mathrm{e}-2$ & $02 \mathrm{e}-2$ & $1.41 \mathrm{e}-2$ & $6.69 \mathrm{e} 9$ & $1.70 \mathrm{e} 9$ & $2.87 \mathrm{e}-1$ & $6 e-2$ & $.55 \mathrm{e}-2$ & e-3 & e10 & $8.27 \mathrm{e} 9$ \\
\hline & & $4.20 \mathrm{e}-3$ & $8.58 \mathrm{e}-3$ & $5.94 \mathrm{e}-2$ & $2 e-2$ & $45 \mathrm{e} 10$ & & $1.08 \mathrm{e}-1$ & & $5.42 \mathrm{e}-2$ & e-3 & $8 \mathrm{e} 11$ & $4.82 \mathrm{e} 10$ \\
\hline JSGA-IIr & 300 & $3.67 \mathrm{e}-3$ & $8.34 \mathrm{e}-3$ & $5.71 \mathrm{e}-2$ & $8.77 \mathrm{e}-3$ & $3.93 \mathrm{e} 10$ & $9.73 \mathrm{e} 9$ & $1.29 \mathrm{e}-1$ & $2.71 \mathrm{e}-2$ & $5.34 \mathrm{e}-2$ & $6.68 \mathrm{e}-3$ & $2.24 \mathrm{e} 11$ & $5.16 \mathrm{e} 10$ \\
\hline SPEA2R & & $3.49 \mathrm{e}-5$ & $1.91 \mathrm{e}-4$ & $4.60 \mathrm{e}-2$ & $16 e-3$ & $5.78 \mathrm{e} 9$ & 9.8 & $3.82 \mathrm{e}-2$ & $1.61 \mathrm{e}-2$ & $4.99 \mathrm{e}-2$ & $2 e-3$ & 7e11 & $2.38 \mathrm{e} 10$ \\
\hline NSGA-IIR & & $1.37 \mathrm{e}-5$ & $7.52 e-5$ & $4.60 \mathrm{e}-2$ & $1.17 \mathrm{e}-3$ & $5.99 \mathrm{e} 9$ & $5.92 \mathrm{e} 8$ & $4.42 \mathrm{e}-2$ & $2.02 \mathrm{e}-2$ & $4.34 \mathrm{e}-2$ & $5.66 e-3$ & $1.47 \mathrm{e} 11$ & $2.65 \mathrm{e} 10$ \\
\hline & & & & & & & & & & & & & \\
\hline NSGA-II & & $8 \mathrm{e}-2$ & $1.81 \mathrm{e}-2$ & $4.81 \mathrm{e}-2$ & e-2 & $7.23 \mathrm{e} 9$ & & $2.91 \mathrm{e}-1$ & le-2 & $3.25 \mathrm{e}-2$ & $9 e-3$ & e10 & $1.04 \mathrm{e} 10$ \\
\hline SPEA2r & 5 & $2.55 \mathrm{e}-3$ & $7.19 e-3$ & $5.99 \mathrm{e}-2$ & $1.02 \mathrm{e}-2$ & $4.50 \mathrm{e} 10$ & $1.15 \mathrm{e} 10$ & $9.18 \mathrm{e}-2$ & $3.32 \mathrm{e}-2$ & $5.58 \mathrm{e}-2$ & $8.17 \mathrm{e}-3$ & $2.65 \mathrm{e} 11$ & $4.30 \mathrm{e} 10$ \\
\hline NSGA-IIr & 500 & $2.52 \mathrm{e}-3$ & $4.81 \mathrm{e}-3$ & $5.85 \mathrm{e}-2$ & & & & & & & & & $7.30 \mathrm{e} 10$ \\
\hline & & $3.49 e-5$ & $1.91 \mathrm{e}-4$ & & & & & & & & & & $1.97 \mathrm{e} 10$ \\
\hline NSGA-IIR & & $0.00 \mathrm{e} 0$ & $0.00 \mathrm{e} 0$ & $4.59 \mathrm{e}-2$ & $1.20 \mathrm{e}-3$ & $6.09 \mathrm{e} 9$ & $4.27 \mathrm{e} 7$ & $2.25 \mathrm{e}-2$ & $1.67 \mathrm{e}-2$ & $4.37 \mathrm{e}-2$ & $5.01 \mathrm{e}-3$ & $1.56 \mathrm{e} 11$ & $2.61 \mathrm{e} 10$ \\
\hline 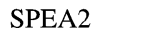 & & & & & & & & & & & & & \\
\hline & & & & $7 e-2$ & & & & & & & & & $9.26 \mathrm{e} 9$ \\
\hline & & $2.02 \mathrm{e}-4$ & $6.77 e-4$ & $5.96 \mathrm{e}-2$ & $4 e-2$ & $4.37 \mathrm{e} 10$ & 1.17 & $5.18 \mathrm{e}-2$ & & $5.76 \mathrm{e}-2$ & $7.44 e-3$ & $3.12 \mathrm{e} 11$ & $6.76 \mathrm{e} 10$ \\
\hline NSGA-IIr & 1000 & $2.09 \mathrm{e}-3$ & $4.90 \mathrm{e}-3$ & $6.05 \mathrm{e}-2$ & $9.55 \mathrm{e}-3$ & $3.92 \mathrm{e} 10$ & $9.68 \mathrm{e} 9$ & $6.70 \mathrm{e}-2$ & $4.04 \mathrm{e}-2$ & $5.56 \mathrm{e}-2$ & $1.25 \mathrm{e}-2$ & 3.17e11 & $8.82 \mathrm{e} 10$ \\
\hline SPEA2R & & $3.38 e-5$ & $1.85 \mathrm{e}-4$ & $4.59 \mathrm{e}-2$ & $1.13 \mathrm{e}-3$ & $6.09 \mathrm{e} 9$ & $3.55 \mathrm{e} 7$ & $9.43 e-3$ & $3.83 \mathrm{e}-3$ & $5.17 \mathrm{e}-2$ & $4.63 \mathrm{e}-3$ & $1.55 \mathrm{e} 11$ & $1.97 \mathrm{e} 10$ \\
\hline NSGA-IIR & & 0.00e0 & $0.00 \mathrm{e} 0$ & $4.58 \mathrm{e}-2$ & $9.56 \mathrm{e}-4$ & $6.09 \mathrm{e} 9$ & $3.55 \mathrm{e} 7$ & $1.27 \mathrm{e}-2$ & $9.33 \mathrm{e}-3$ & $4.34 \mathrm{e}-2$ & $5.07 \mathrm{e}-3$ & $1.69 \mathrm{e} 11$ & $1.84 \mathrm{e} 10$ \\
\hline
\end{tabular}

\section{APPENDIX \\ PERFORMANCE METRICS}

Table IV presents the mean values and the standard deviations for the three metrics obtained in the M-JPEG encoder and JPEG decoder case studies. Best values are shown in bold.

\section{ACKNOWLEDGMENT}

The authors thank Prof. X. Yao and the anonymous reviewers for their valuable feedback, which helped to improve the quality of this paper.

\section{REFERENCES}

[1] K. Keutzer, S. Malik, R. Newton, J. Rabaey, and A. Sangiovanni-Vincentelli, "System level design: Orthogonalization of concerns and platform-based design," IEEE Trans. Computer-Aided Design, vol. 19, no. 12, pp. 1523-1543, 2000

[2] F. Balarin, Y. Watanabe, H. Hsieh, L. Lavagno, C. Passerone, and A. Sangiovanni-Vincentelli, "Metropolis: An integrated electronic system design environment," IEEE Computer, vol. 36, no. 4, pp. 45-52, 2003.
[3] A. Stammermann, L. Kruse, W. Nebel, A. Pratsch, E. Schmidt, M. Schulte, and A. Schulz, "System level optimization and design space exploration for low power," in Proc. Int. Symp. Systems Synthesis, Oct. 2001, pp. 142-146.

[4] T. Givargis, F. Vahid, and J. Henkel, "Instruction-based system-level power evaluation of system-on-a-chip peripheral cores," IEEE Trans. VLSI Syst., vol. 10, pp. 856-863, 2002.

[5] J. E. Coffland and A. D. Pimentel. A software framework for ef ficient system-level performance evaluation of embedded systems. presented at Proc. ACM Symp. Applied Computing. [Online]. Available: http://sesamesim.sourceforge.net/

[6] A. D. Pimentel, C. Erbas, and S. Polstra, "A systematic approach to exploring embedded systems architectures at multiple abstraction levels," IEEE Trans. Comput., vol. 55, no. 2, pp. 99-112, Feb. 2006.

[7] A. D. Pimentel, P. Lieverse, P. van der Wolf, L. O. Hertzberger, and E. F." Deprettere, "Exploring embedded-systems architectures with Artemis," IEEE Computer, pp. 57-63, Nov. 2001

[8] C. Erbas, S. Cerav-Erbas, and A. D. Pimentel, "A multiobjective optimization model for exploring multiprocessor mappings of process networks," in Proc. Int. Conf. Hardware/Software Codesign and System Synthesis, Oct. 2003, pp. 182-187.

[9] J. D. Schaffer, "Multiple objective optimization with vector evaluated genetic algorithms," in Proc. Int. Conf. Genetic Algorithms, 1985, pp. 93-100.

[10] C. M. Fonseca and P. J. Flemming, "Genetic algorithms for multiobjective optimization: Formulation, discussion and generalization," in Proc. Int. Conf. Genetic Algorithms, 1993, pp. 416-423. 
[11] N. Srinivas and K. Deb, "Multiobjective optimization using nondominated sorting in genetic algorithms," Evol. Comput., vol. 2, no. 3, pp. 221-248, 1994.

[12] K. Deb, S. Agrawal, A. Pratap, and T. Meyarivan, "A fast elitist nondominated sorting genetic algorithm for multi-objective optimization: NSGA-II," in Parallel Problem Solving from Nature-PPSN $V I$, M. Schoenauer, K. Deb, G. Rudolph, X. Yao, E. Lutton, J. Merelo, and H. Schwefel, Eds. Berlin, Germany: Springer, 2000, pp. 849-858.

[13] E. Zitzler, M. Laumanns, and L. Thiele, "SPEA2: Improving the strength pareto evolutionary algorithm for multiobjective optimization," in Evolutionary Methods for Design, Optimization, and Control, K. Giannakoglou, D. Tsahalis, J. Periaux, K. Papailiou, and T. Fogarty, Eds. Barcelona, Spain: CIMNE, 2002, pp. 95-100.

[14] M. T. Jensen, "Reducing the run-time complexity of multi-objective EAs: The NSGA-II and other algorithms," IEEE Trans. Evol. Comput., vol. 7, pp. 503-515, 2003.

[15] G. G. Yen and L. Haiming, "Dynamic multiobjective evolutionary algorithm: Adaptive cell-based rank and density estimation," IEEE Trans. Evol. Comput., vol. 7, pp. 253-274, 2003.

[16] H. Lu and G. G. Yen, "Rank-density-based multiobjective genetic algorithm and benchmark test function study," IEEE Trans. Evol. Comput., vol. 7, pp. 325-343, 2003.

[17] T. Blickle, J. Teich, and L. Thiele, "System-level synthesis using evolutionary algorithms," Design Autom. Embed. Syst., vol. 3, no. 1, pp. 23-58, 1998.

[18] R. P. Dick and N. K. Jha, "MOGAC: A multiobjective genetic algorithm for hardware-software co-synthesis of distributed embedded systems," IEEE Trans. Computer-Aided Design, pp. 920-935, Oct. 1998.

[19] L. Thiele, S. Chakraborty, M. Gries, and S. Künzli, "A framework for evaluating design tradeoffs in packet processing architectures," in Proc. Design Automation Conf., Jun. 2002, pp. 880-885.

[20] T. Givargis, F. Vahid, and J. Henkel, "System-level exploration for pareto-optimal configurations in parameterized system-on-a-chip," IEEE Trans. VLSI Syst., vol. 10, pp. 416-422, 2002.

[21] T. Givargis and F. Vahid, "Platune: A tuning framework for system-on-a-chip platforms," IEEE Trans. Computer-Aided Design, vol. 21, pp. 1317-1327, 2002.

[22] G. Ascia, V. Catania, and M. Palesi, "A GA-based design space exploration framework for parameterized system-on-a-chip platforms," IEEE Trans. Evol. Comput., vol. 8, pp. 329-346, 2004.

[23] R. Szymanek, F. Catthoor, and K. Kuchcinski, "Time-energy design space exploration for multi-layer memory architectures," in Proc. Design, Automation Test Eur., Feb. 2004, pp. 10318-10323.

[24] G. Alpaydin, S. Balkir, and G. Dundar, "An evolutionary approach to automatic synthesis of high-performance analog integrated circuits," IEEE Trans. Evol. Comput., vol. 7, pp. 240-252, 2003.

[25] M. S. Bright and T. Arslan, "Synthesis of low-power DSP systems using a genetic algorithm," IEEE Trans. Evol. Comput., vol. 5, pp. 27-40, 2001.

[26] J. C. Gallagher, S. Vigraham, and G. Kramer, "A family of compact genetic algorithms for intrinsic evolvable hardware," IEEE Trans. Evol. Comput., vol. 8, pp. 111-126, 2004.

[27] G. Kahn, "The semantics of a simple language for parallel programming," in Proc. IFIP Congress, 1974, pp. 471-475.

[28] R. E. Steuer and E. U. Choo, "An interactive weighted Tchebycheff procedure for multiple objective programming," Math. Program., vol. 26, pp. 326-344, 1983.

[29] M. Ehrgott, Multicriteria Optimization, ser. Lecture Notes in Economics and Mathematical Systems. Heidelberg, Germany: Springer-Verlag, 2000.

[30] R. E. Steuer, "An overview in graphs of multiple objective programming," in Proc. Int. Conf. Evolutionary Multi-Criterion Optimization, vol. 1993, LNCS, E. Zitzler, K. Deb, L. Thiele, C. A. Coello Coello, and D. Corne, Eds., 2001, pp. 41-51.

[31] C. A. Coello Coello, "Guest editorial: Special issue on evolutionary multiobjective optimization," IEEE Trans. Evol. Comput., vol. 7, pp. 97-99, 2003.

[32] C. A. Coello Coello, D. A. Van Veldhuizen, and G. B. Lamont, Evolutionary Algorithms for Solving Multi-Objective Problems. New York: Kluwer Academic, 2002.

[33] D. Beasley, D. Bull, and R. Martin, "An overview of genetic algorithms: Part 1, fundamentals," Univ. Comput., vol. 15, no. 2, pp. 58-69, 1993.

[34] M. Laumanns, E. Zitzler, and L. Thiele, "A unified model for multi-objective evolutionary algorithms with elitism," in Proc. Congr. Evolutionary Computation, 2000, pp. 46-53.
[35] E. Zitzler, K. Deb, and L. Thiele, "Comparison of multiobjective evolutionary algorithms: Empirical results," Evol. Comput., vol. 8, no. 2, pp. 173-195, 2000.

[36] E. Zitzler, "Evolutionary algorithms for multiobjective optimization: methods and applications," Ph.D. dissertation, Swiss Federal Inst. of Technology, Zurich, 1999.

[37] G. Rudolph, "Evolutionary search under partially ordered fitness sets," in Proc. Int. NAISO Congr. Information Science Innovations, M. F. Sebaaly, Ed., Millet, Canada, 2001, pp. 818-822.

[38] D. E. Goldberg, Genetic Algorithms in Search, Optimization, and Machine Learning. Reading, MA: Addison-Wesley, 1989.

[39] W. Spears, "Crossover or mutation?," in Proc. Workshop Foundations Genetic Algorithms, Jul. 1992, pp. 221-237.

[40] G. Syswerda, "Uniform crossover in genetic algorithms," in Proc. Int Conf. Genetic Algorithms, 1989, pp. 2-9.

[41] K. Deb, Multi-Objective Optimization Using Evolutionary Algorithms. New York: Wiley, 2001.

[42] J. Knowles and D. Corne, "On metrics for comparing nondominated sets," Proc. Congr. Evolutionary Computation, pp. 711-716, May 2002.

[43] A. D. Pimentel, S. Polstra, F. Terpstra, A. W. van Halderen, J. E. Coffland, and L. O. Hertzberger, "Toward efficient design space exploration of heterogeneous embedded media systems," in Embedded Processor Design Challenges: Systems, Architectures, Modeling, and Simulation. ser. LNCS, E. Deprettere, J. Teich, and S. Vassiliadis, Eds. Berlin, Germany: Springer-Verlag, 2002, vol. 2268, pp. 57-73.

[44] E. A. de Kock, "Multiprocessor mapping of process networks: A JPEG decoding case study," in Proc. Int. Symp. System Synthesis, Oct. 2002, pp. $68-73$.

[45] S. Bleuler, M. Laumanns, L. Thiele, and E. Zitzler, "PISA-A platform and programming language independent interface for search algorithms," in Evolutionary Multi-Criterion Optimization (EMO 2003). ser. LNCS, C. M. Fonseca, P. J. Fleming, E. Zitzler, K. Deb, and L. Thiele, Eds. Berlin, Germany: Springer-Verlag, 2003, vol. 2632, pp. 494-508.

[46] P. Lieverse, T. Stefanov, P. van der Wolf, and E. F. Deprettere, "System level design with spade: An M-JPEG case study," in Proc. Int. Conf. Computer Aided Design, Nov. 2001, pp. 31-38.

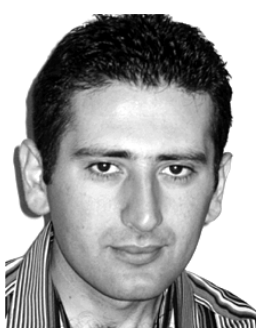

Cagkan Erbas (S'99) received the B.Sc. degree in electrical engineering from Middle East Technical University, Ankara, Turkey, and the M.Sc. degree in computer engineering from Ege University, Izmir, Turkey. He is currently working towards the Ph.D. degree in computer science at the University of Amsterdam, The Netherlands.

His research interests include embedded systems, hardware/software codesign, multiobjective search algorithms, and metaheuristics.

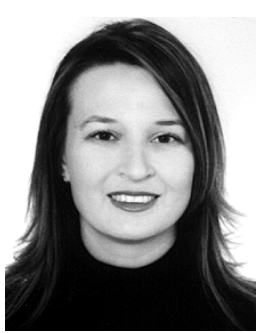

Selin Cerav-Erbas received the B.Sc. degree from Bilkent University, Ankara, Turkey, the M.Sc. degree from Georgia Institute of Technology, Atlanta, both in industrial engineering, and the Ph.D. degree in mathematics from RWTH Aachen, Germany.

She is currently a Postdoctoral Researcher in the School of Management, Université Catholique de Louvain, Belgium. Her research interests include Internet traffic engineering, multiobjective search algorithms, and metaheuristics.

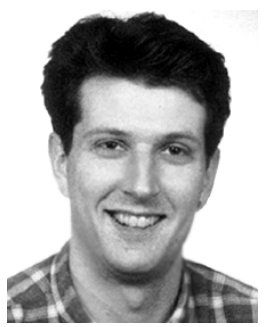

Andy D. Pimentel received the M.Sc. and Ph.D. degrees in computer science from the University of Amsterdam, Amsterdam, The Netherlands.

$\mathrm{He}$ is currently an Assistant Professor in the Informatics Institute, University of Amsterdam. $\mathrm{He}$ is member of the European Network of Excellence on High-Performance Embedded Architecture and Compilation. His research interests include computer architecture, computer architecture modeling and simulation, system-level design, design space exploration, performance analysis, embedded systems,

and parallel computing.

Prof. Pimentel is a member of the IEEE Computer Society. 